(C) 2020. This manuscript version is made available under the CC-BY-NC-ND 4.0 license http://creativecommons.org/licenses/by-nc-nd/4.0/ 


\title{
Adaptive Energy Shaping Control of Robotic Needle Insertion
}

\author{
Enrico Franco* 1, Timothy Brown ${ }^{1}$, Alessandro Astolfi ${ }^{2,3}$, Ferdinando Rodriguez y Baena ${ }^{1}$ \\ ${ }^{1}$ Mechanical Engineering Department, Imperial College London, UK \\ ${ }^{2}$ Electrical and Electronic Engineering Department, Imperial College London, UK \\ ${ }^{3}$ Dipartimento di Ingegneria Civile e Ingegneria Informatica, Università di Roma "Tor Vergata" \\ Rome, 00133, Italy \\ * Corresponding author: ef1311@imperial.ac.uk
}

\begin{abstract}
This work studies the control of a pneumatic actuator for needle insertion in soft tissue without using axial rotation or additional needle supports. Employing a simplified rigid-link model description of an axial-symmetric tip needle supported at the base, two energy shaping controllers are proposed. The friction forces of the pneumatic actuator are compensated adaptively and the stability conditions for the closed-loop equilibrium are discussed. The controllers are compared by means of simulations and experiments on two different silicone rubber phantoms. The results indicate that the proposed controllers effectively compensate the actuator's friction, which is comparable to the insertion forces for the chosen pneumatic actuators. The first controller only depends on the actuator's position thus it achieves the prescribed insertion depth but results in a larger tip rotation and corresponding deflection. The second controller also accounts for the rotation of the needle tip on the bending plane, which can consequently be reduced by over $70 \%$ for this specific system. This is achieved by modulating the actuator force and, in case of harder phantoms, by automatically limiting the insertion depth.
\end{abstract}

Keywords: Underactuated systems, Force Control, Adaptive Control, Pneumatic Systems, Medical Robotics.

\section{Introduction}

Needle insertion is common to several minimally invasive surgical procedures, including biopsies and brachytherapy. Although in clinical practice the majority of needle insertions are still conducted manually, the benefits of employing robotic systems in terms of repeatability and accuracy have motivated a considerable amount of research. Robotic systems that assist or automatically perform needle insertions are deemed particularly beneficial for image-guided surgery, for which access to the patient is severely restricted by the imaging equipment [1,2]. A particularly prominent class of robotic systems for needle insertion employs the so-called remote centre of motion (RCM) structure [3]. This configuration allows reorienting the needle while maintaining the position of the insertion point thus reducing the size of the robot. In addition, RCM robots keep their mechanism and actuators away from the patient to avoid collisions and interferences [4]. Besides incorrect needle placement, needle deflection is one of the main causes of error in percutaneous interventions and it is associated with an increased risk of damaging healthy tissue. In conventional manual procedures the error is typically corrected by removing the needle and by repeating the insertion, thus causing patient discomfort and increasing the duration of the clinical procedure [5,6]. Needle deflection can occur at any point during the insertion, depending on the needle geometry and on the forces acting on it. Considering that insertion forces typically peak during the initial puncture [7] and that the free length of the needle is then at its maximum, deflections are particularly likely to occur in this condition. Slender needles, such as those used in biopsy, can also exhibit buckling, which might permanently damage the needle and could compromise the clinical procedure. Taking inspiration from nature, a number of design principles that can reduce the risk of needle deflection have been highlighted in [8]. However, their implementation is often limited by manufacturing capabilities. Various actuation strategies have also been proposed to control needle deflection [9]. One of the most popular approaches is the controlled axial rotation of bevel-tip needles [10]: since the asymmetry of the bevel results in a lateral force on the tip, the deflection can be modulated by rotating the needle around its axis. This approach can be used for the purpose of needle steering, thus achieving curved insertion paths to avoid obstacles. Conversely, following a straight insertion path requires a constant axial 
rotation, which can damage soft tissues [11]. To overcome this problem different strategies have been devised, including: a programmable needle tip consisting of multiple segments pushed independently at the back [12]; a multi-segment needle consisting of nitinol wires [13]; a steerable needle actuated by heat transmitted with an optic fibre [14]; concentric tube robots used as steerable needles [15]. In spite of these remarkable advances, conventional metallic needles remain ubiquitous in clinical practice, thus the study of control strategies for the insertion of standard needles is an active area of research [16]. Important results in this sense include: sliding-mode control algorithms [17,18]; modelbased optimal control of flexible needles [19,20]; an optimized path planning procedure for insertions in multi layered tissues [21]; an adaptive output-feedback controller [22]; an integrator backstepping approach to reduce needle deflection in 3D [11]. Notably, these approaches aim to influence the needle deflection as the insertion progresses by means of axial rotation of bevel-tip needles. However, a recurring problem in clinical practice corresponds to performing a straight needle insertion employing multi-bevel needles or needles with axial-symmetric tip [23]. Compared to the insertion of bevel tip needles, this problem has remained relatively unexplored. In particular, axial rotation has almost exclusively been employed for bevel-tip needles, while its effectiveness for axial-symmetric tip needles is scarcely documented. Finally, while needle deflection can be reduced with the aid of a needle guide, this approach might not be viable in some procedures due to space restrictions or to risks of collision with the patient, and it might not be appropriate for some RCM robot designs. Thus the investigation of an alternative solution is of particular practical relevance.

In this work two new adaptive nonlinear controllers for the insertion of a needle with axialsymmetric tip employing a linear pneumatic actuator are designed by using an energy shaping methodology. We have chosen this control approach since it is widely applicable to underactuated mechanical systems and since it provides a convenient interpretation of the control action in terms of the energy of the closed-loop system. In summary the main contributions of this work include the following points.

1. Two nonlinear control laws are designed, and a direct physical interpretation is provided in terms of mechanical energy of the closed-loop system. The controllers extend our preliminary work [24] by including an adaptive observer designed with the Immersion and Invariance methodology $[25,26]$ to compensate the unknown friction forces of the pneumatic actuator

2. The corresponding stability conditions are studied with a Lyapunov approach and tuning guidelines for the controller parameters are discussed.

3. Simulations and experiments using a needle with axial-symmetric tip and two silicone rubber phantoms of different hardness representative of two types of soft tissues are presented. The results demonstrate that both controllers can compensate the actuator's friction. The first controller achieves the desired insertion depth with both phantoms but at the cost of larger tip rotations. Instead, the second controller limits the tip rotation by modulating the actuator force and, in case of harder phantoms, by automatically limiting the insertion depth.

The rest of the paper is organized as follows. Section 2 presents the problem formulation, Section 3 the controller design, Section 4 presents the experimental results and Section 5 the conclusions.

\section{Problem Formulation}

\subsection{System overview}

The system considered in this work employs a single linear actuator that applies a force along the neutral axis of the needle, see Figure 1. This configuration is common to several surgical robots for percutaneous intervention [3,4] and it can also be part of a teleoperation setup [27]. The use of a single actuator allows to reduce the complexity of the mechanism, its weight and size, and its cost, but results in an underactuated system which is more difficult to control, since typically not all degrees of freedom (DOFs) can be regulated to the prescribed value. In general, underactuation can result from actuator failure or from structural compliance [28], thus investigating the proposed system could also be beneficial to the study of robotic needle insertion in more complex setups. We employ pneumatic actuation because of its suitability to force control and its compatibility to medical imaging modalities [1]. The system consists of a double-acting plastic pneumatic cylinder (AC111-707-501, 
IPS Inc.) powered by two proportional valves (Tecno Basic, Hoerbiger). Each valve contains an onboard pressure sensor and regulates the output pressure with an internal PID control loop. Figure 2 shows the system schematic. The piston position $q_{1}$ is measured with an optical encoder (EM1-300, US Digital). The insertion force consists of the axial component $F$ parallel to the actuator, which is measured with a force sensor (FSG15N1A, Honeywell), and of the lateral component $F^{\prime}$ orthogonal to the actuator. A $18 \mathrm{G}$ coaxial titanium needle with an internal electromagnetic sensor (Aurora $5 \mathrm{DOF}$ sensor with 0.5-mm diameter and 8-mm length, part number 610061, Northern Digital Inc.) is mounted on the piston with a specially designed adapter so that the base of the needle rests on the force sensor. In this study the angle $q_{2}$ between the needle tip and the axial direction $x$ is measured with an electromagnetic tracking system (Aurora, Northern Digital Inc.) that has a root mean square (RMS) accuracy of 0.2 degrees [29]. In clinical practice this could be achieved with medical imaging modalities, such as MRI, using a standard MRI-safe needle and a bespoke localization algorithm, such as [30]. The clinical setup would also require a bespoke MRI-safe force sensor, such as [31].

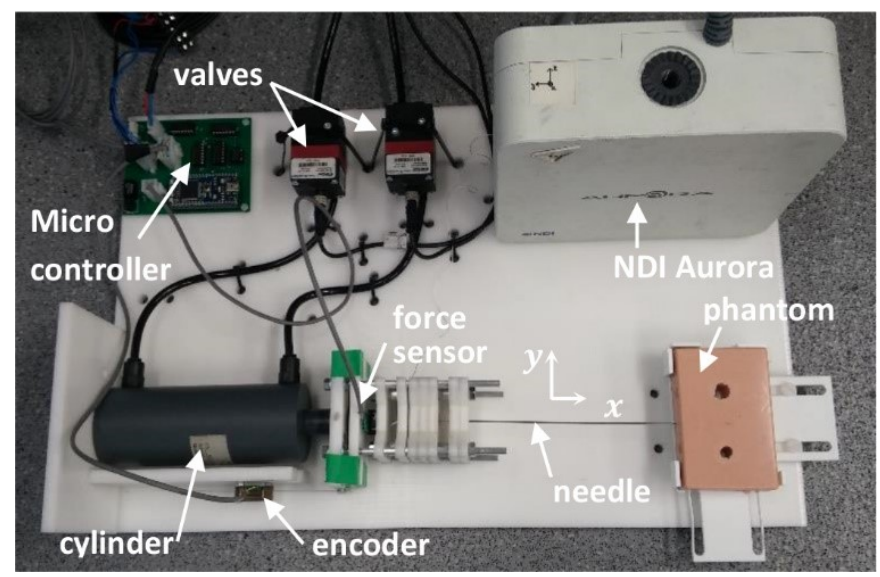

Figure 1. Photograph of the experimental setup showing the main components of the system.

\subsection{System model}

For controller design purposes the needle is treated as a cantilever beam and is modelled as a planar rigid-link system with a virtual elastic joint of stiffness $k_{t}$ located at a distance $l$ from the tip (see Figure 2). This approach is based on the pseudo-rigid-body model methodology [32] which provides a simplified method for modelling the force/deflection relationship in compliant mechanisms. Our prior work [28] indicates that this approach is sufficiently accurate for controller design purposes, provided the deflection of the cantilever beam corresponds to the first mode shape. The length $l$ of the virtual link and the elastic constant $k_{t}$ of the virtual joint are defined as

$$
\begin{gathered}
l=\gamma_{\theta} L, \\
k_{t}=\gamma_{\theta} k_{\theta} E I / L .
\end{gathered}
$$

The parameters $\gamma_{\theta}, k_{\theta}$ depend on the loading conditions of the cantilever beam and are defined to ensure kineto-static equivalence between the flexible beam and the rigid-link model. Their value can be computed with an optimization routine that approximates the closed-form solution of the elliptic integral corresponding to the deflection path (see [33] for further details). The terms $E$ and $I$ are the Young's modulus and the area moment of inertia of the beam, and $L$ is its length. The equations of motion for the coordinate axes $x$ and $y$ are thus

$$
\begin{gathered}
(M+m) \ddot{q}_{1}-m l \sin \left(q_{2}\right) \ddot{q}_{2}-m l \cos \left(q_{2}\right) \dot{q}_{2}^{2}= \\
P_{1} A_{1}-P_{0} A_{0}-F_{c} \tanh \left(\dot{q}_{1}\right)-F_{s}-F_{v} \dot{q}_{1}-F, \\
m l^{2} \ddot{q}_{2}-m l \sin \left(q_{2}\right) \ddot{q}_{1}=-k_{t} q_{2}-c_{v} \dot{q}_{2}+F l \sin \left(q_{2}\right)+F^{\prime} l \cos \left(q_{2}\right) .
\end{gathered}
$$

The coefficient $c_{v}>0$ represents the mechanical damping of the cantilever beam and it is typically obtained experimentally [34]. A simplified friction model is employed for the actuator: tanh ( $)$ is a continuous approximation of the sign function; the Coulomb friction $F_{c}$, the static friction $F_{s}$, and the 
viscous friction $F_{v}$ of the pneumatic actuator are typically unknown. The pressure $P_{1}$ in the left chamber of the cylinder of area $A_{1}$ is the control input, and the pressure $P_{0}$ in the right chamber of area $A_{0}$ is kept constant for simplicity. Considering the high flow rate and the fast response of the valves $(10 \mathrm{~ms})$ and the comparatively low insertion speed in percutaneous interventions (i.e. less than $10 \mathrm{~mm} / \mathrm{s}$ [7]), the pressure dynamics is not accounted for in this work. This approximation introduces a small modelling error compared to the uncertainty associated to the friction forces of the pneumatic cylinder. The mass of all translating parts (i.e. piston, force sensor, adapter) is $M$, and the mass of the passive link $m$ is concentrated at the tip. The model has 2 DOFs but only the translation $q_{1}$ is actuated. Assuming negligible twist, which is reasonable if the axial rotation is not actuated and if there are no external twisting moments, the angle $q_{2}$ determines the needle deflection $\sigma=l \sin \left(q_{2}\right)$ on any plane of bending, thus (2) represents the system dynamics in 3D. Introducing a needle guide to reduce the tip deflection equates to reducing the free length $L$ of the needle and consequently the length $l$ of the rigid link, and to increasing the stiffness $k_{t}$ of the virtual joint according to (1). As a result, the proposed model and the resulting controllers remain valid even in the presence of a needle guide provided the system parameters are appropriately modified.

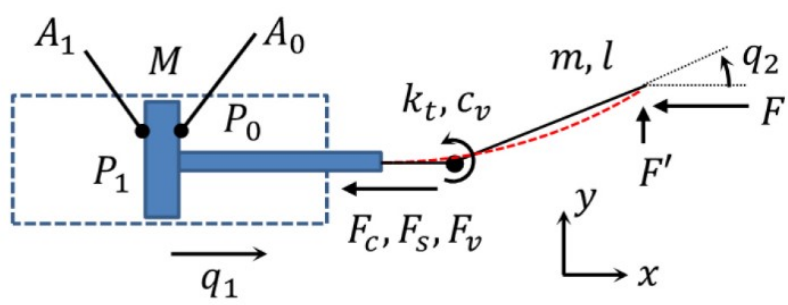

Figure 2. Schematic of the actuator and of the rigid-link model of the needle on the bending plane.

\section{Main results}

This section presents the controller design for the underactuated system (2) with the aim to approach the prescribed insertion depth $q_{1}^{*}$ in soft tissues with arbitrarily small tip rotations $q_{2}^{*}$ and corresponding deflections. Thus the regulation goal is $\left(q_{1}, q_{2}\right)=\left(q_{1}^{*}, q_{2}^{*}\right)$ with $\left|q_{2}^{*}\right| \ll 1$.

\subsection{Adaptive friction compensation}

The first step in the controller design aims at compensating the friction of the pneumatic actuator, which is typically unknown and can degrade the system performance. An adaptive law is constructed by employing the Immersion and Invariance methodology $[25,26]$ for the general case of $n$ additive linearly parameterized disturbances. Thus the following assumptions are introduced.

Assumption 1: the sum of the friction forces acting on the piston is defined as $\rho=f^{T} \zeta$, where $f\left(q_{1}\right)$ is a vector of $n$ known continuous functions, and $\zeta$ is a vector of $n$ unknown constant parameters.

Assumption 2: all remaining model parameters in (1) and (2) are exactly known. The axial force $F>$ 0 , its derivative $\dot{F}$, and the states $q_{1}, q_{2}, \dot{q}_{1}, \dot{q}_{2}$ are measurable and bounded, with $\left|q_{2}\right|<\pi / 2$ and $q_{1}>$ 0 by construction. Instead, the lateral force $F^{\prime}$ is assumed to be zero for controller design purposes. The effect of a lateral force $F^{\prime} \neq 0$ on the system dynamics is discussed separately in Remark 2 . Finally, the needle twist is assumed to be zero.

In practice Assumption 1 is verified by choosing an appropriate friction model (see Section 2.2). In addition, Assumption 2 is typically verified considering the following points: the axial force $F$ is directed against the actuator; the insertion is stopped if $\left|q_{2}\right| \geq \pi / 2$ to avoid permanent deformation of the needle; in this study the states $q_{1}, q_{2}$ and the axial force $F$ are directly measured (see Section 2.1 ); the setup does not include axial rotation and the phantom is stationary, thus twist is null. The design of the adaptive nonlinear observer is detailed in the following proposition.

Proposition 1: Consider system (2) under Assumptions 1 and 2. Let the vector of estimation errors for the unknown parameters be $z=\tilde{\zeta}-\zeta$, where $\tilde{\zeta}=(\hat{\zeta}+\beta)$ is the adaptive estimate of $\zeta$, consisting 
of the estimator state $\hat{\zeta}$ and of the state-dependent function $\beta$ (see $[25,26]$ for further details on this notation). Consider the adaptive law

$$
\begin{gathered}
\dot{\hat{\zeta}}=\alpha f\left(P_{1} A_{1}-P_{0} A_{0}-F \cos \left(q_{2}\right)^{2}+m l \cos \left(q_{2}\right) \dot{q}_{2}^{2}\right) \\
-\alpha f\left(\frac{1}{l}\left(k_{t} q_{2}+c_{v} \dot{q}_{2}\right) \sin \left(q_{2}\right)+f^{T}(\hat{\zeta}+\beta)\right)-\alpha \Phi m \sin \left(2 q_{2}\right) \dot{q}_{2}, \\
\beta=-\alpha \Phi\left(M+m \cos \left(q_{2}\right)^{2}\right),
\end{gathered}
$$

where $f=d \Phi / d \dot{q}_{1}$ and $\alpha>0$ is a tuning parameter. Then the scalar quantity $f^{T} z$ converges to zero exponentially.

Proof: Computing the time derivative of $z$ and substituting $\ddot{q}_{1}$ obtained from (2.a) and (2.b) yields

$$
\begin{gathered}
\dot{z}=\dot{\zeta}+\frac{d \beta}{d q_{2}} \dot{q}_{2}+\frac{d \beta}{d \dot{q}_{1}} \frac{\left(P_{1} A_{1}-P_{0} A_{0}+m l \cos \left(q_{2}\right) \dot{q}_{2}^{2}\right)}{M+m \cos \left(q_{2}\right)^{2}} \\
-\frac{d \beta}{d \dot{q}_{1}} \frac{\left(\left(k_{t} q_{2}+c_{v} \dot{q}_{2}\right) \sin \left(q_{2}\right) / l+f^{T}(\hat{\zeta}+\beta-z)-F^{\prime} \sin \left(q_{2}\right) \cos \left(q_{2}\right)+F \cos \left(q_{2}\right)^{2}\right)}{M+m \cos \left(q_{2}\right)^{2}} .
\end{gathered}
$$

Defining the Lyapunov function candidate $W=\frac{1}{2} z^{T} z$, computing its time derivative and substituting (3) and (4) where $F^{\prime}=0$ by hypothesis (see Assumption 2) gives

$$
\dot{W}=-\alpha z^{T} f f^{T} z<0 .
$$

Thus $f^{T} Z$ converges to zero exponentially for all $\alpha>0$

For the friction model in (2) we have $\zeta^{T}=\left[\begin{array}{lll}F_{c} & F_{s} & F_{v}\end{array}\right], f^{T}=\left[\tanh \left(\dot{q}_{1}\right) \quad 1 \quad \dot{q}_{1}\right], \quad \rho=$ $F_{c} \tanh \left(\dot{q}_{1}\right)+F_{S}+F_{v} \dot{q}_{1}$, and $\Phi^{\mathrm{T}}=\left[\log \left(\cosh \left(\dot{q}_{1}\right)\right) \quad \dot{q}_{1} \quad \dot{q}_{1}^{2} / 2\right]$. While Proposition 1 ensures that the cumulative estimate of the friction forces converges to $\rho$, the individual estimates of $F_{c}, F_{s}, F_{v}$ might not converge to the actual values of the corresponding parameters. In theory, an unknown and constant lateral force $F^{\prime}$ could be treated in a similar way as the friction forces by including in $f$ a further term dependent on $q_{2}$. The study of this aspect goes beyond the scope of this paper and it is part of our future work.

\subsection{Controller 1}

Without loss of generality, the open-loop dynamics (2) in the presence of the unknown actuator friction $\rho$ and of the known external forces $\delta$ is expressed in port-Hamiltonian form

$$
\left[\begin{array}{c}
\dot{q} \\
\dot{p}
\end{array}\right]=\left[\begin{array}{cc}
0 & I \\
-I & -D
\end{array}\right]\left[\begin{array}{l}
\nabla_{q} H \\
\nabla_{p} H
\end{array}\right]+\left[\begin{array}{l}
0 \\
G
\end{array}\right]\left(v-\rho-P_{0} A_{0}\right)-\left[\begin{array}{l}
0 \\
\delta
\end{array}\right]
$$

The system states are the position $q \in \mathbb{R}^{2}$ and the momenta $p=\mathcal{M} \dot{q}$. The Hamiltonian is defined as $H=T(q, p)+V(q)$, with kinetic energy $T(q, p)=\frac{1}{2} p^{T} \mathcal{M}^{-1} p$, inertia matrix $\mathcal{M}(q)=\mathcal{M}^{T}(q)>$ 0 , and potential energy $V(q)$. The damping matrix is $D=D^{T}>0$ and $I$ is the identity matrix. The remaining terms are the control input $v$, the input matrix $G \in \mathbb{R}^{2 \times 1}$, and the back pressure $P_{0}$. The expressions of $\mathcal{M}, V, D, G$ and $\delta$, which depends on $F$ and $F^{\prime}$, are provided in the Appendix. The two symbols $\nabla_{q}\left({ }^{\cdot}\right)$ and $\nabla_{p}\left({ }^{\cdot}\right)$ represents the gradient with respect to $q$ and $p$ of a scalar function. The control law $v$ is designed according to the Interconnection and Damping Assignment Passivity Based Control methodology (IDA-PBC) [35], which aims to conveniently shape the kinetic energy and the potential energy of the system in closed loop. A different energy shaping approach was employed in our prior work [28] and relies on a more restrictive set of assumptions, which are not verified for system (2). The IDA-PBC methodology consists in defining the control law in such a way that the resulting closed-loop dynamics is representative of a new mechanical system with total energy $H_{d}$

$$
\left[\begin{array}{c}
\dot{q} \\
\dot{p}
\end{array}\right]=\left[\begin{array}{cc}
0 & \mathcal{M}^{-1} \mathcal{M}_{d} \\
-\mathcal{M}_{d} \mathcal{M}^{-1} & J_{2}-G k_{v} G^{T}-D \mathcal{M}^{-1} \mathcal{M}_{d}
\end{array}\right]\left[\begin{array}{l}
\nabla_{q} H_{d} \\
\nabla_{p} H_{d}
\end{array}\right]
$$


where $J_{2}=-J_{2}^{T}$ is a free matrix, and $k_{v}=k_{v}^{T}>0$ is a tuning parameter. The new total energy is defined here as $H_{d}=\frac{1}{2} p^{T} \mathcal{M}_{d}^{-1} p+V_{d}+\Psi$ such that it has a strict minimizer at the desired equilibrium $(q, p)=\left(q^{*}, 0\right)$ by enforcing the constraints $\nabla_{q} H_{d}\left(q^{*}, 0\right)=0$ and $\nabla_{q}^{2} H_{d}\left(q^{*}, 0\right)>0$. This is an essential step in the energy shaping procedure for the purpose of achieving the regulation goal $(q, p)=\left(q^{*}, 0\right)$. The inertia matrix $\mathcal{M}_{d}=\mathcal{M}_{d}^{T}>0$ and the potential energy $V_{d}$ are the main design parameters, while $\Psi$ is the work of the external forces $F$ and $F^{\prime}$ in the closed-loop system. Equating (6) and (7) and defining the pseudo inverse of $G$ as $G^{\dagger}=\left(G^{T} G\right)^{-1} G^{T}$ the expression of the energy shaping control law for the case of known external forces $\delta$ becomes

$$
\begin{gathered}
v=v_{e s}+v_{d i}+\left(f^{T}(\hat{\zeta}+\beta)+P_{0} A_{0}\right) / A_{1}, \\
v_{e s}=G^{\dagger}\left(\nabla_{q} H-\mathcal{M}_{d} \mathcal{M}^{-1} \nabla_{q} H_{d}+J_{2} \mathcal{M}_{d}^{-1} p+\delta\right), \\
v_{d i}=-k_{v} G^{T} \nabla_{p} H_{d} .
\end{gathered}
$$

In order for the control law (8) to be implementable, the matrices $\mathcal{M}_{d}, J_{2}$, and the potential energy $V_{d}$ should satisfy the partial-differential-equations (PDEs)

$$
\begin{gathered}
G^{\perp}\left(\nabla_{q}\left(p^{T} \mathcal{M}^{-1} p\right)-\mathcal{M}_{d} \mathcal{M}^{-1} \nabla_{q}\left(p^{T} \mathcal{M}_{d}^{-1} p\right)+2 J_{2} \mathcal{M}_{d}^{-1} p\right)=0 \\
G^{\perp}\left(\nabla_{q} V-\mathcal{M}_{d} \mathcal{M}^{-1}\left(\nabla_{q} V_{d}\right)\right)=0, \\
G^{\perp}\left(\delta-\mathcal{M}_{d} \mathcal{M}^{-1} \nabla_{q} \Psi\right)=0,
\end{gathered}
$$

where $G^{\perp}$ is a full-rank left annihilator of $G$, that is $G^{\perp} G=0$ and $\operatorname{rank}\left\{G^{\perp}\right\}=1$. Since $\nabla_{q} \Psi$ depends on the external forces $\delta$, this contribution is not accounted for within $V_{d}$ but is instead treated separately in (9.c), similarly to [36]. Setting $\mathcal{M}_{d}=k_{m} \mathcal{M}$, with $k_{m}>0$ and $J_{2}=0$, verifies (9.a), while (9.b), (9.c) become linear PDEs. Solving (9) for system (2) with $\mathcal{M}_{d}=k_{m} \mathcal{M}$ yields

$$
\begin{aligned}
& V_{d}=\frac{k_{t}}{2 k_{m}} q_{2}^{2}+\frac{k_{p}}{2 k_{m}} h\left(q_{1}^{*}-q_{1}\right)^{2}, \\
& \Psi=\frac{l}{k_{m}}\left(F \cos \left(q_{2}\right)-F^{\prime} \sin \left(q_{2}\right)\right),
\end{aligned}
$$

where the function $h(\cdot)$ should be chosen to enforce the minimizer conditions. Selecting $h\left(q_{1}^{*}-q_{1}\right)=\left(q_{1}^{*}-q_{1}\right)$ and computing (8), where $P_{1}=v$ and $\hat{\zeta}$ and $\beta$ are defined in (3), results in the control law corresponding to Controller 1

$$
v=\frac{1}{A_{1}}\left(k_{p}\left(q_{1}^{*}-q_{1}\right)-\frac{k_{v}}{k_{m}} \dot{q}_{1}+P_{0} A_{0}+f^{T}(\hat{\zeta}+\beta)+F\right) .
$$

Proposition 2: Consider the system (2) under Assumptions 1 and 2 in closed-loop with the control law (11). In particular, assume $F<k_{t} / l, F^{\prime}=0$, and $|\dot{F}|<\kappa\left(\dot{q}^{T} G\right)^{2}$ for some $\kappa>0$. Then $q^{*}=$ $\left(q_{1}^{*}, 0\right)$ is a strict minimizer of $H_{d}$ and a locally asymptotically stable equilibrium of the closed-loop system provided $2 \alpha k_{v}>1$ and $k_{v}>2 \kappa l k_{m}$.

Proof: Computing the minimizer conditions at $q^{*}=\left(q_{1}^{*}, 0\right)$, which corresponds to the straight needle and to the prescribed insertion depth $q_{1}^{*}$, yields

$$
\begin{gathered}
\nabla_{q} H_{d}\left(q^{*}, 0\right)=\left[\begin{array}{c}
0 \\
-F^{\prime} l / k_{m}
\end{array}\right]=0, \\
\nabla_{q}^{2} H_{d}\left(q^{*}, 0\right)=k_{p}\left(k_{t}-F l\right) /\left(m k_{m}^{2}\right)>0,
\end{gathered}
$$

which are verified for $F^{\prime}=0$ and $F<k_{t} / l$, thus proving the first claim.

To prove the second claim note that, since $\Psi$ is positive definite for all $F>0$ and all $\left|q_{2}\right|<\frac{\pi}{2}$ provided $F^{\prime}=0$, then also $H_{d}$ is positive definite and radially unbounded thus it qualifies as a Lyapunov function candidate. Since (11) contains the adaptive estimate $(\hat{\zeta}+\beta)$, the estimation error 
$f^{T} z$ propagates to (6) and to (7) through the input matrix $G$. Thus the stability analysis should account for the error dynamics. Defining the new Lyapunov function candidate $W^{\prime}=H_{d}+W$, computing its time derivative, and substituting (5) and (7), where $\mathcal{M}_{d}=k_{m} \mathcal{M}$ yields

$$
\dot{W}^{\prime}=-\dot{q}^{T}\left(\frac{D k_{m}+G k_{v} G^{T}}{k_{m}^{2}}\right) \dot{q}+\dot{F} l \frac{\cos \left(q_{2}\right)}{k_{m}}+\dot{q}^{T} G \frac{f^{T} z}{k_{m}}-\alpha z^{T} f f^{T} z .
$$

Refactoring the terms in (13) gives

$$
\dot{W}^{\prime}=-\left[\begin{array}{ll}
\dot{q}^{T} & z^{T} f
\end{array}\right]\left[\begin{array}{cc}
\frac{2 D k_{m}+G k_{v} G^{T}}{2 k_{m}^{2}} & \frac{G}{2 k_{m}} \\
\frac{G^{T}}{2 k_{m}} & \alpha
\end{array}\right]\left[\begin{array}{c}
\dot{q} \\
f^{T} z
\end{array}\right]-\frac{k_{v}}{2 k_{m}^{2}}\left(\dot{q}^{T} G\right)^{2}+\dot{F} l \frac{\cos \left(q_{2}\right)}{k_{m}} .
$$

Computing the Schur complement in (14) shows that $\dot{W}^{\prime} \leq 0$ provided $\alpha\left(2 D k_{m}+G k_{v} G^{T}\right)-$ $G G^{T} / 2>0$, which is verified if $2 \alpha k_{v} \geq 1$, and provided $k_{v}\left(\dot{q}^{T} G\right)^{2}>2 \dot{F} l k_{m}$, where $k_{v}$ and $\alpha$ are tuning parameters. In addition, recalling that $|\dot{F}|<\kappa\left(\dot{q}^{T} G\right)^{2}$ by hypothesis, the second inequality yields $k_{v}>2 \kappa l k_{m}$. It follows from (14) that $\dot{q} \in \mathcal{L}^{2} \cap \mathcal{L}^{\infty}$ and $\dot{p} \in \mathcal{L}^{\infty}$, thus $\dot{q}$ converges to zero asymptotically according to Barbalat's Lemma, while $f^{T} z$ converges to zero from Proposition 1. Computing (7) at the equilibrium by setting $\dot{p}=p=0$ yields $\nabla_{q} H_{d}\left(q^{*}, 0\right)=0$ which implies $q=$ $q^{*}$ from (12.a). Consequently, the equilibrium $q^{*}$ is locally asymptotically stable

\subsection{Controller 2}

For comparative purposes, a nonlinear control law is constructed by using a partial feedback linearization, that is

$$
\begin{gathered}
P_{1}=\frac{M+m \cos \left(q_{2}\right)^{2}}{A_{1}} v+\frac{\left(k_{t} q_{2}+c_{v} \dot{q}_{2}\right) \sin \left(q_{2}\right)}{l A_{1}} \\
+\frac{1}{A_{1}}\left(-m l \dot{q}_{2}^{2} \cos \left(q_{2}\right)+P_{0} A_{0}+f^{T}(\hat{\zeta}+\beta)\right) .
\end{gathered}
$$

Substituting (15) into (2) yields again (6) but with different parameters $\mathcal{M}^{\prime}, G^{\prime}, D^{\prime}, V^{\prime}$ defined in the Appendix. Solving (9.b) and (9.c) with $\mathcal{M}_{d}^{\prime}=k_{m} \mathcal{M}^{\prime}$ yields in this case

$$
\begin{gathered}
V_{d}^{\prime}=\frac{k_{t}}{2 m l^{2} k_{m}} q_{2}^{2}+\frac{k_{p}}{2 k_{m}} h\left(\left(q_{1}^{*}-q_{1}\right) l+\cos \left(q_{2}\right)\right)^{2}, \\
\Psi^{\prime}=\frac{1}{m l k_{m}}\left(F \cos \left(q_{2}\right)-F^{\prime} \sin \left(q_{2}\right)\right) .
\end{gathered}
$$

Choosing $h(\eta)=\log (\eta)$, where $\eta=\left(q_{1}^{*}-q_{1}\right) l+\cos \left(q_{2}\right)$, verifies the minimizer conditions at $q^{*}=$ $\left(q_{1}^{*}, 0\right)$ provided $F^{\prime}=0$. Instead, setting $h(\eta)=\eta$ as in (11) would not verify condition (12.a). Substituting (16) in (8) and into (15) yields the control law corresponding to Controller 2

$$
\begin{gathered}
P_{1}=\frac{P_{0} A_{0}}{A_{1}}+\frac{\left(k_{t} q_{2}+c_{v} \dot{q}_{2}\right)}{A_{1} l} \sin \left(q_{2}\right)-\frac{m l \dot{q}_{2}^{2} \cos \left(q_{2}\right)}{A_{1}}+\frac{f^{T}(\hat{\zeta}+\beta)}{A_{1}}-\frac{F^{\prime}}{2 A_{1}} \sin \left(2 q_{2}\right) \\
+k_{p} l \Delta \frac{\log \left(\left(q_{1}^{*}-q_{1}\right) l+\cos \left(q_{2}\right)\right)}{A_{1}\left(\left(q_{1}^{*}-q_{1}\right) l+\cos \left(q_{2}\right)\right)}-\frac{k_{v}}{k_{m}} \Delta \frac{\left(l \dot{q}_{1}+\sin \left(q_{2}\right) \dot{q}_{2}\right)}{l A_{1}}+\frac{F}{A_{1}} \cos \left(q_{2}\right)^{2},
\end{gathered}
$$

where $\Delta=m \cos \left(q_{2}\right)^{2}+M$, and $\hat{\zeta}, \beta$ are defined in (3).

Proposition 3: Consider the system (2), under Assumptions 1 and 2 in closed-loop with the control law (17). In particular, assume $F<k_{t} / l, F^{\prime}=0$, and $|\dot{F}|<\kappa\left(\dot{q}^{T} G^{\prime}\right)^{2}$ for some $\kappa>0$. Then $q^{*}=$ $\left(q_{1}^{*}, 0\right)$ is a strict minimizer of $H_{d}^{\prime}$ and a locally asymptotically stable equilibrium of the closed-loop system provided $2 k_{v} \alpha M^{2}>1$ and $k_{v}>2 \kappa k_{m} /(m l)$. 
Proof: To prove the first claim we compute the minimizer conditions from (16) recalling that $F^{\prime}=0$ (see Assumption 2), which yields again (12). The proof of the second claim follows closely that of Proposition 2. Computing the time derivative of the Lyapunov function candidate $W^{\prime \prime}=H_{d}^{\prime}+W$, where we define $H_{d}^{\prime}=\frac{1}{2} p^{T} \mathcal{M}_{d}^{\prime-1} p+V_{d}^{\prime}+\Psi^{\prime}$, yields in this case

$$
\dot{W}^{\prime \prime}=-\left[\begin{array}{ll}
\dot{q}^{T} & z^{T} f
\end{array}\right]\left[\begin{array}{cc}
\frac{2 D k_{m}+G^{\prime} k_{v} G^{\prime T}}{2 k_{m}^{2}} & \frac{G^{\prime}}{2 k_{m} \Delta} \\
\frac{G^{\prime T}}{2 k_{m} \Delta} & \alpha
\end{array}\right]\left[\begin{array}{c}
\dot{q} \\
f^{T}{ }_{z}
\end{array}\right]-\frac{k_{v}}{2 k_{m}^{2}}\left(\dot{q}^{T} G^{\prime}\right)^{2}+\dot{F} \frac{\cos \left(q_{2}\right)}{m l k_{m}} .
$$

Computing the Schur complement in (18) yields $\dot{W}^{\prime \prime} \leq 0$ provided $\alpha\left(2 D k_{m}+G^{\prime} k_{v} G^{\prime T}\right)-$ $G^{\prime} G^{\prime T} / 2 \Delta^{2}>0$ and $k_{v} m l\left(\dot{q}^{T} G^{\prime}\right)^{2}>2 \dot{F} k_{m}$. Since $\Delta \geq M$, the first inequality is verified for all $2 k_{v} \alpha M^{2}>1$, while recalling that $|\dot{F}|<\kappa\left(\dot{q}^{T} G^{\prime}\right)^{2}$ by hypothesis the second inequality yields $k_{v} m l>2 k_{m} \kappa$. Using similar arguments as those in Proposition 2, it follows that the equilibrium $q^{*}$ is locally asymptotically stable

Remark 1: Note that Controller 1 does not account for the flexibility of the needle thus it treats the system (2) as a single DOF model. Consequently, the tuning guidelines in Proposition 2 do not depend on the states $q_{2}$ and $\dot{q}_{2}$. In particular, the assumption $|\dot{F}|<\kappa\left(\dot{q}^{T} G\right)^{2}$ corresponds to accounting for nonlinear viscous friction between needle and tissue. Instead, Controller 2 accounts for the states $q_{2}$ and $\dot{q}_{2}$, which also appear in the tuning guidelines provided in Proposition 3. Note however that, if $q_{2} \cong 0$, the assumption $|\dot{F}|<\kappa\left(\dot{q}^{T} G^{\prime}\right)^{2}$ in Proposition 3 is equivalent to $|\dot{F}|<$ $\kappa\left(\dot{q}^{T} G\right)^{2}$ in Proposition 2. In addition, (18) also depends on the mass of the piston thus the stability conditions translate into higher values of $k_{v}$ and $\alpha$ if $M<1$. For both controllers, the prescribed equilibrium becomes unstable if $F>k_{t} / l$. Computing $k_{t}$ from (1) with $k_{\theta}=2.4$ from [33], the value $k_{t} / l$ corresponds, with good approximation, to the first Euler-Bernoulli buckling load for a cantilever beam with tip force, that is $F=(\pi / 2)^{2} E I / l^{2}$. Thus the condition $F \geq k_{t} / l$ corresponds to the elastic instability of the Euler-Bernoulli beam. In this case, comparing the minimizer condition $\nabla_{q} H_{d}\left(q^{*}, 0\right)=0$ for Controller 1 and the corresponding condition $\nabla_{q} H_{d}^{\prime}\left(q^{*}, 0\right)=0$ for Controller 2 reveals the existence of additional equilibria even if $F^{\prime}=0$, that is

$$
\begin{gathered}
\nabla_{q} H_{d}(q, 0)=\left[\begin{array}{c}
\frac{k_{p}}{k_{m}}\left(q_{1}-q_{1}^{*}\right) \\
\frac{k_{t}}{k_{m}} q_{2}-\frac{F l}{k_{m}} \sin \left(q_{2}\right)-\frac{F^{\prime} l}{k_{m}} \cos \left(q_{2}\right)
\end{array}\right], \\
\nabla_{q} H_{d}^{\prime}(q, 0)=\left[\begin{array}{c}
-\frac{k_{p} l}{k_{m}} \frac{\log (\eta)}{\eta} \\
-\frac{F}{m l k_{m}} \sin \left(q_{2}\right)-\frac{F^{\prime}}{m l k_{m}} \cos \left(q_{2}\right)+\frac{k_{t}}{m l^{2} k_{m}} q_{2}-\frac{k_{p}}{k_{m}} \sin \left(q_{2}\right) \frac{\log (\eta)}{\eta}
\end{array}\right],
\end{gathered}
$$

where $\eta=\left(\left(q_{1}^{*}-q_{1}\right) l+\cos \left(q_{2}\right)\right)$. For small tip rotations $q_{2} \cong 0$ the trigonometric functions in the second element of (19.a) can be approximated with truncated Taylor series. Equating the resulting expression to zero yields the quadratic equation $\left(\left(k_{t}-F l\right)+F l q_{2}^{2} / 6\right) q_{2}-F^{\prime} l\left(1-q_{2}^{2} / 2\right)=0$. Solving the former equation for $F^{\prime}=0$ gives the straight-needle equilibrium $q_{2}=0$ and the additional equilibria $q_{2}= \pm \sqrt{6\left(1-k_{t} / F l\right)}$, which only exist if $F>k_{t} / l$ and correspond to the tip rotation according to the first buckling mode. Comparing the first element of (19.a) and (19.b) shows that with Controller 1 the insertion depth $q_{1}=q_{1}^{*}$ remains a strict minimizer of $H_{d}$ even if $q_{2}^{*} \neq 0$. Conversely, if $q_{2}^{*} \neq 0$ the strict minimizer of $H_{d}^{\prime}$ is located at a shorter insertion depth $q_{1}^{\prime}$ which corresponds to $\eta=1$. In other words, with Controller 2 the insertion might be automatically stopped at $q_{1}^{\prime}$ if the tip rotation is not zero, where $q_{1}^{\prime}=q_{1}^{*}+\left(\cos \left(q_{2}\right)-1\right) / l$ (see Section 4). Equating the 
second element of (19.b) to zero and approximating the trigonometric functions with truncated Taylor series yields $\left(\left(k_{t}-F l-k_{p} m l^{2} \log (\eta) / \eta\right)+F l q_{2}^{2} / 6\right) q_{2}-F^{\prime} l\left(1-q_{2}^{2} / 2\right)=0$. Thus the straightneedle equilibrium $q_{2}=0$ is still present if $F^{\prime}=0$ and corresponds to $q_{1}=q_{1}^{*}$. Instead, an analytical expression of the additional equilibria cannot be provided in this case since $q_{2}$ is also contained within $\eta$. In case $q_{2} \ll 1$, we can approximate $\eta \cong\left(\left(q_{1}^{*}-q_{1}\right) l+1\right)$ thus the additional equilibria would coincide in practice with those computed from (19.a). Note finally that employing a needle guide would result in a smaller $l^{\prime}<l$ and a larger $k_{t}^{\prime}>k_{t}$ according to (1), thus the equilibrium would remain stable for larger axial forces $F<k_{t}^{\prime} / l^{\prime}$, where $k_{t}^{\prime} / l^{\prime}>k_{t} / l$.

Remark 2: Note that Controller 1 does not account for the lateral force $F^{\prime}$. If the latter is also omitted in the observer (3) two additional terms appear in the Lyapunov function time derivative (14), that is

$$
\dot{W}^{\prime *}=\dot{W}^{\prime}+F^{\prime} \frac{\cos \left(q_{2}\right)\left((m+M) \dot{q}_{2}+m l \sin \left(q_{2}\right) \dot{q}_{1}\right)}{k_{m} l\left(M+m \cos \left(q_{2}\right)^{2}\right)}-\frac{1}{2} \alpha z^{T} f f^{T} F^{\prime} \sin \left(2 q_{2}\right) .
$$

Introducing the Young's inequality $\frac{1}{2} \alpha z^{T} f f^{T} F^{\prime} \sin \left(2 q_{2}\right) \leq \frac{1}{8} \alpha\left|z^{T} f\right|^{2}+\frac{1}{2}\left|f^{T} F^{\prime} \sin \left(2 q_{2}\right)\right|^{2}$ and computing the corresponding sufficient stability conditions in proximity of the equilibrium yields

$$
\begin{gathered}
\frac{7}{8} \alpha\left(2 D k_{m}+G k_{v} G^{T}\right)-\frac{1}{2} G G^{T}>0, \\
k_{v} \dot{q}_{1}^{2}>2 l k_{m} \kappa \dot{q}_{1}^{2}+2 F^{\prime} k_{m}\left(\frac{1}{l}\left(\frac{m}{M}+1\right) \dot{q}_{2}+\frac{m}{M} \sin \left(q_{2}\right) \dot{q}_{1}\right)+k_{m}^{2}\left|f^{T} F^{\prime} \sin \left(2 q_{2}\right)\right|^{2},
\end{gathered}
$$

which are more stringent than those expressed in Proposition 2. Instead, Controller 2 does include the term $F^{\prime}$. However, in case $F^{\prime}$ is omitted from (17) and from (3), its effect on the Lyapunov derivative is the same as in (20) resulting in more stringent stability conditions than those expressed in Proposition 3. This indicates that neglecting large lateral forces can have a destabilizing effect on the system. In our simulations and experiments the lateral force $F^{\prime}$ is small compared to the axial force $F$ and to the friction forces (see Section 4) thus the tuning parameters have been chosen according to Proposition 2 and Proposition 3. This approach might not be appropriate for larger lateral forces which might occur within the context of deeper needle insertions. This scenario shall be investigated in more detail as part of our future work.

Remark 3: Substituting (17) into (3) yields the explicit expression of the adaptive law

$$
\dot{\hat{\zeta}}=\alpha f \Delta\left(k_{p} l \frac{\log \left(\left(q_{1}^{*}-q_{1}\right) l+\cos \left(q_{2}\right)\right)}{\left(\left(q_{1}^{*}-q_{1}\right) l+\cos \left(q_{2}\right)\right)}-\frac{k_{v}}{k_{m}} \frac{\left(l \dot{q}_{1}+\sin \left(q_{2}\right) \dot{q}_{2}\right)}{l}\right)-\alpha \Phi m \sin \left(2 q_{2}\right) \dot{q}_{2},
$$

which is nonlinear and depends on the position error of the piston, on the needle tip rotation $q_{2}$, and on the velocities $\dot{q}_{1}$ and $\dot{q}_{2}$. Substituting (11) into (3) yields a similar expression to (22), which in addition also contains the terms $k_{t}$ and $c_{v}$. In summary, (22) shows that combining Controller 2 with the nonlinear observer (3) results in a nonlinear control law that requires full state feedback (see Assumption 2), which is also the case for Controller 1. Thus employing the nonlinear observer (3) goes beyond industry-standard controllers, such as the PID, in which the integral action would only account for the position error of the actuator. In addition, the proposed controllers allow for a physical interpretation in terms of mechanical energy of the closed-loop system.

\section{Experimental results}

\subsection{Simulations}

The system (2) has been simulated in Matlab using the parameters $m=0.001 \mathrm{Kg} ; M=$ $0.05 \mathrm{Kg} ; A_{1}=A_{0}=1 \mathrm{~cm}^{2} ; l=180 \mathrm{~mm} ; k_{t}=0.130 \mathrm{Nm} ; c_{v}=1 ; P_{0}=0$. For illustrative purposes, the friction forces of the actuator are set as $F_{c}=F_{S}=F_{v}=0.05 \mathrm{~N}$, and the axial force is modelled as

$$
F=\left(c_{0}+c_{1} y_{t i p}+c_{2} \tanh \left(c_{3} \dot{y}_{t i p}\right)\right) k_{t} / l
$$


where $y_{\text {tip }}=q_{1}+l\left(\cos \left(q_{2}\right)-1\right)$ is the needle tip position and $\dot{y}_{\text {tip }}=\dot{q}_{1}-\sin \left(q_{2}\right) l \dot{q}_{2}$ is the corresponding velocity, and $c_{0}, c_{1}, c_{2}, c_{3}$ are scalar coefficients representing respectively the fracture energy of the tissue, its elasticity, and its viscous friction, while $c_{3}$ is a scaling factor. The values $c_{0}=$ $0.75, c_{1}=2.5, c_{2}=0.25, c_{3}=100$, which refer to an ideal phantom, have been used for the simulations in this section. Further simulation results with different parameters that correspond to the experimental setup are presented in Section 4.2. The tuning parameters are $k_{p}=2 ; k_{v}=15$ and $\alpha=$ 50 for Controller 1, denoting the control law (11) with the observer (3), and $k_{p}=20 ; k_{m}=1 ; k_{v}=$ 3 and $\alpha=50$ for Controller 2, denoting the control law (17) with the observer (3).
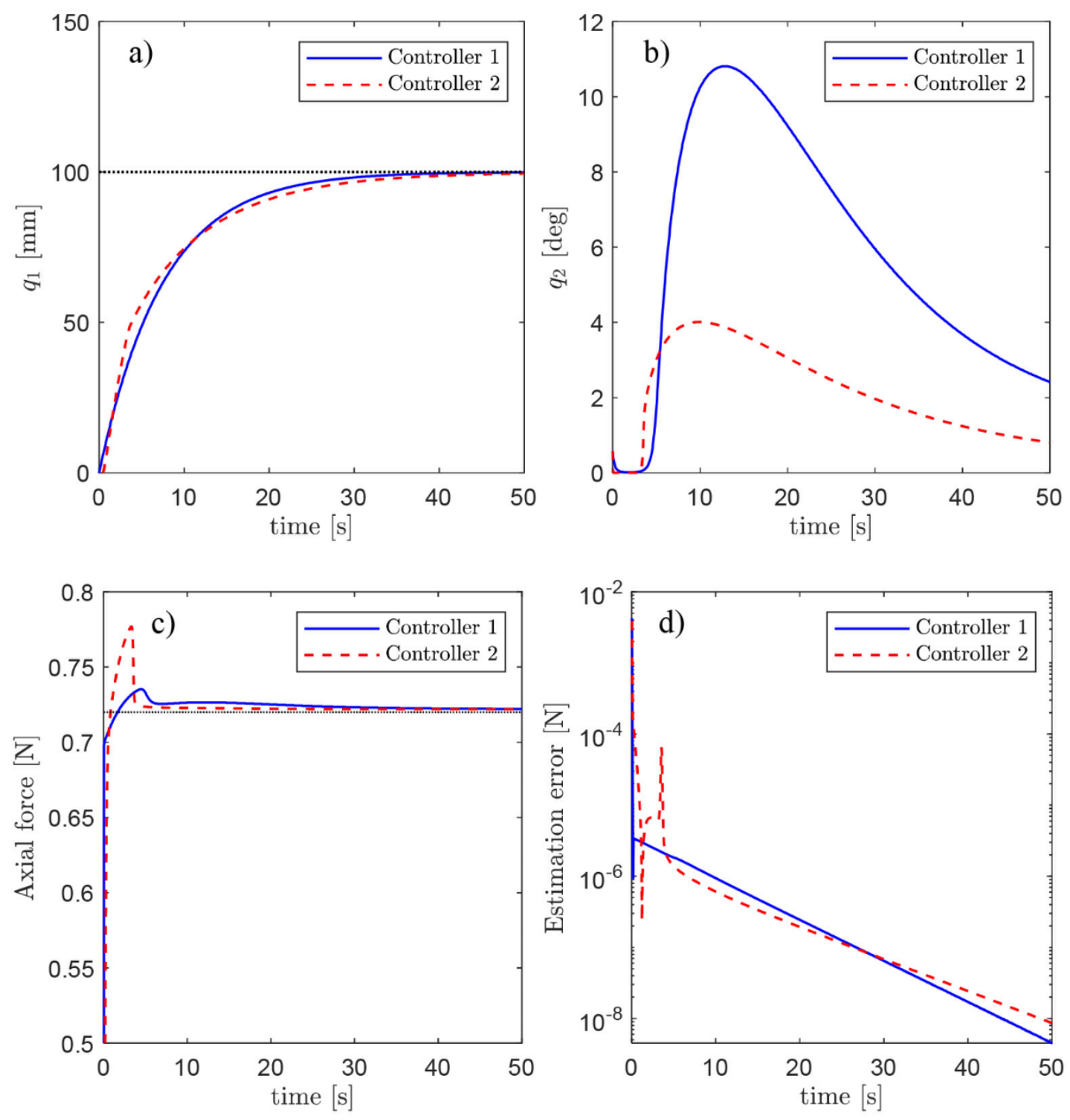

Figure 3. Simulation results without lateral force: piston position (a); tip rotation (b); axial force (c); cumulative estimation error on a logarithmic scale (d). Controller 1 denotes the control law (11) with the observer (3), while Controller 2 denotes the control law (17) with the observer (3).

The simulation results in Figure 3 show that the Controller 1 drives the needle to the desired depth rapidly but with a noticeable tip rotation. The insertion speed is approximately $2 \mathrm{~mm} / \mathrm{s}$, which is comparatively low in clinical practice. Simulation results and experimental results for faster insertions representative of percutaneous interventions are presented in Section 4.2. Conversely, the Controller 2 allows reaching the desired insertion depth with a smaller tip rotation. The corresponding tip deflection according to the model (1) and (2) is $\sigma=l \sin \left(q_{2}\right)$. Figure 3.c shows that, for the Controller 2, the axial force increases at first but then drops rapidly as soon as the needle starts deflecting. Figure 3.d shows that in both cases the estimation errors converge to zero. Figure 4 shows the effect of a small and known lateral force $F^{\prime}=F \in$ that becomes active at time 30 seconds, where 
$\epsilon=0.001$ can be interpreted as an asymmetry in the phantom. With Controller 1 the piston still reaches the prescribed position but with a larger tip rotation. Conversely, Controller 2 stops the insertion before reaching the prescribed depth as discussed in Remark 1. It must be noted that the sudden change in the tip rotation at time 30 seconds is due to the step change of $\epsilon$ at that instant. This scenario is valuable from a theoretical point of view, but it is not necessarily clinically representative.
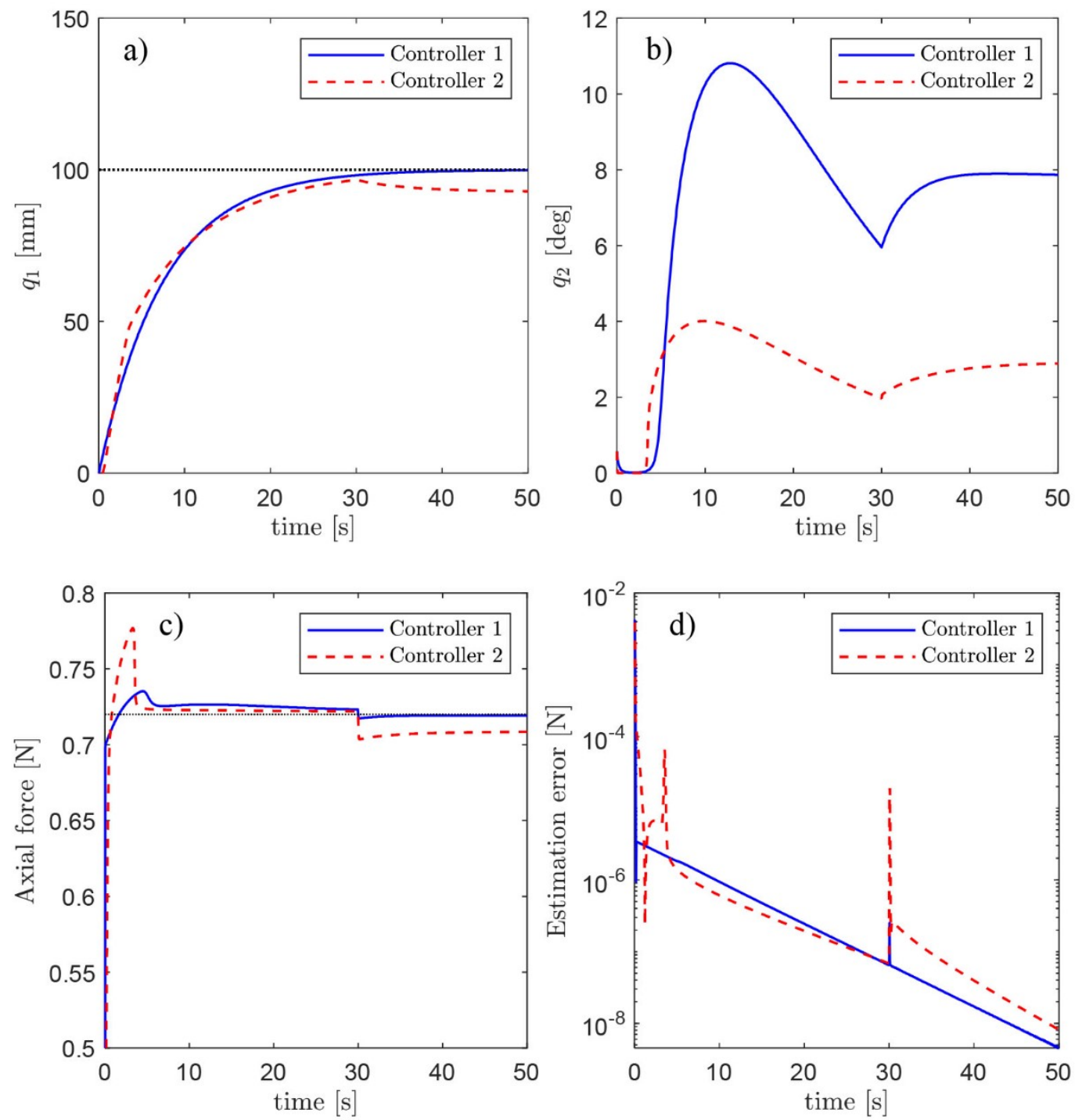

Figure 4. Simulation results with a known lateral force $F^{\prime}=F \epsilon$ acting on the needle after time $t=30$ seconds: piston position (a); tip rotation (b); axial force (c); cumulative estimation error on a logarithmic scale (d). Controller 1 denotes the control law (11) with the observer (3), while Controller 2 denotes the control law (17) with the observer (3).

Figure 5 shows that setting $\alpha=0$, which corresponds to our preliminary work without friction compensation [24], the actuator friction is not compensated and the regulation goal is not achieved even though no lateral force is present. In particular, with Controller 1 the piston position stops at approximately $75 \mathrm{~mm}$, while with Controller 2 the piston does not move at all employing the current tuning $k_{p}=20$. This occurs because the proportional gain $k_{p}$, which modulates the control input depending on the position of the piston, is multiplied by the terms $l$ and $\Delta$ in (17) resulting in a smaller actuator force that is not sufficient to overcome the actuator friction. Employing $k_{p}=1000$ with Controller 2 the piston position converges to a similar value to that achieved using Controller 1 . In either case the tip rotation $q_{2}$ converges to zero however the insertion is not completed.

Figure 6 shows the simulation results for a baseline controller consisting of a PID algorithm with feed-forward compensation of the axial force $F$ and of the back pressure $P_{0}$ resulting in the 
control law $v=\left(K_{p}\left(q_{1}^{*}-q_{1}\right)-K_{v} \dot{q}_{1}+K_{i} \int\left(q_{1}^{*}-q_{1}\right) d t+P_{0} A_{0}+F\right) / A_{1}$. The PID represents a valuable term of comparison since it is widely employed in surgical robotics and it constitutes the basis of recent controllers for robotic needle insertion [17]. The tuning parameters have been chosen iteratively as $K_{p}=4, K_{v}=15$, which are comparable to $k_{p}, k_{v}$ used for Controller 1 , and $K_{i}=0.1$. The system response is qualitatively similar to that of Controller 1 however the tip rotation reaches higher values. Decreasing $K_{p}$ or increasing $K_{v}$ reduces the maximum tip rotation $q_{2}$, but $q_{1}$ shows a noticeable overshoot, which is typically undesirable in clinical practice. Instead, increasing $K_{i}$ causes oscillations on $q_{1}$ and $q_{2}$.
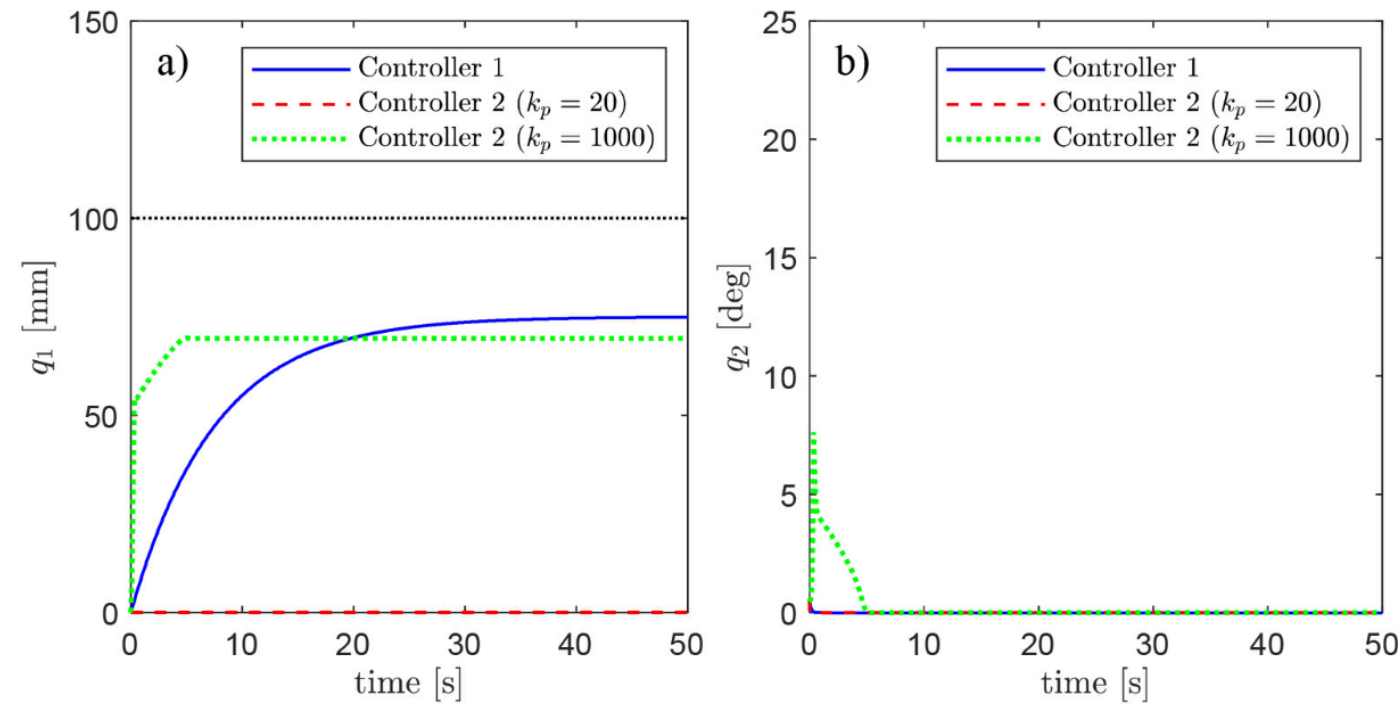

Figure 5. Simulation results without lateral force and without friction compensation: piston position (a); tip rotation (b).
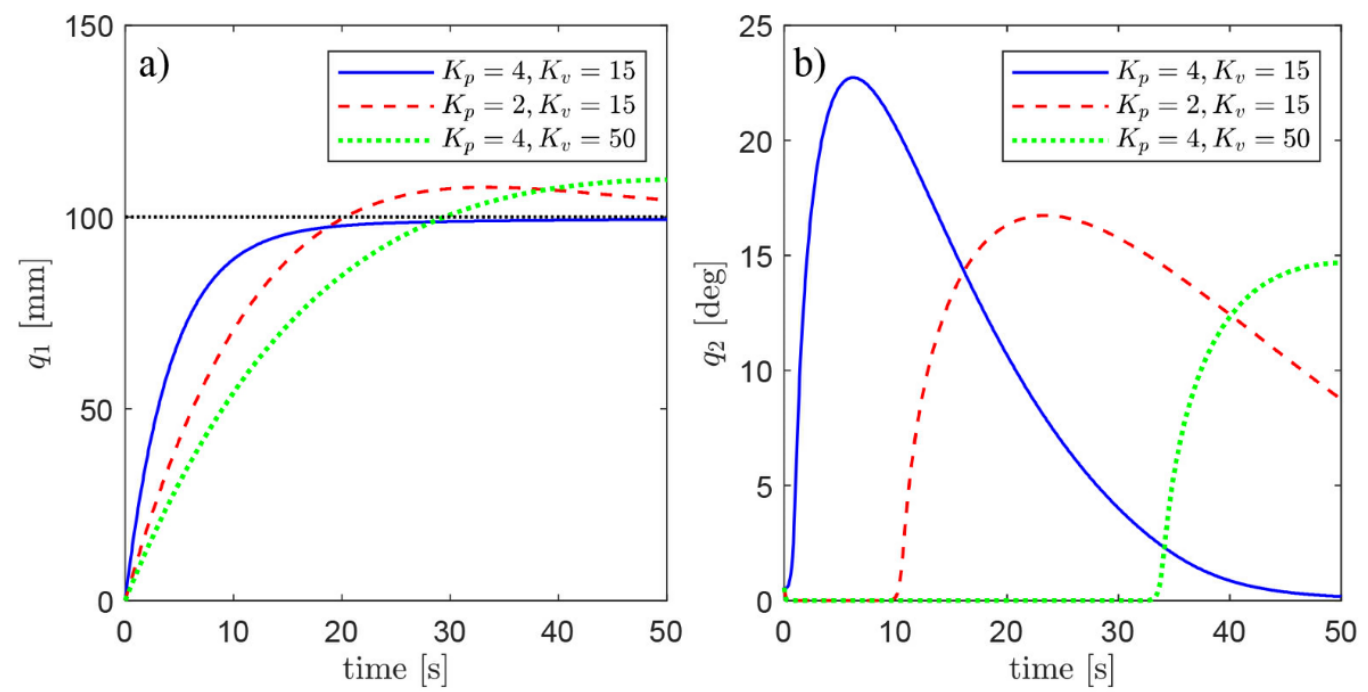

Figure 6. Simulation results for baseline PID controller without lateral force: piston position (a); tip rotation $(b)$.

\subsection{Experiments}

Needle insertion experiments have been conducted using a $18 \mathrm{G}$ coaxial titanium needle with axial-symmetric tip ( $1.3 \mathrm{~mm} \mathrm{OD}, 0.8 \mathrm{~mm} \mathrm{ID,} 150 \mathrm{~mm}$ long) to minimise interference with the tracking system, using two silicone rubber phantoms (PlatSil GEL-10, Polytek) of different hardness (Shore A $=1$ for the softer phantom; Shore A = 10 for the harder phantom). Silicone rubber has been widely used to investigate needle insertions [37] since it has mechanical properties similar to those of soft tissues. In particular, Shore $A=10$ is representative of liver tissues [38], while Shore $A=1$ is 
representative of breast tissues [39]. Nevertheless, silicone rubber phantoms represent only a simplified approximation of physiological tissues, which are characterized by complex internal structures, such as blood vessels. The tests have been repeated three times for each phantom with different puncture locations providing comparable results. The maximum deviation between trials at insertion completed (time $=3$ seconds for illustrative purposes) is $0.1 \mathrm{~mm}$ for $q_{1}$ and 0.5 degrees for $q_{2}$ with Controller 1, and $1.9 \mathrm{~mm}$ for $q_{1}$ and 0.9 degrees for $q_{2}$ with Controller 2.
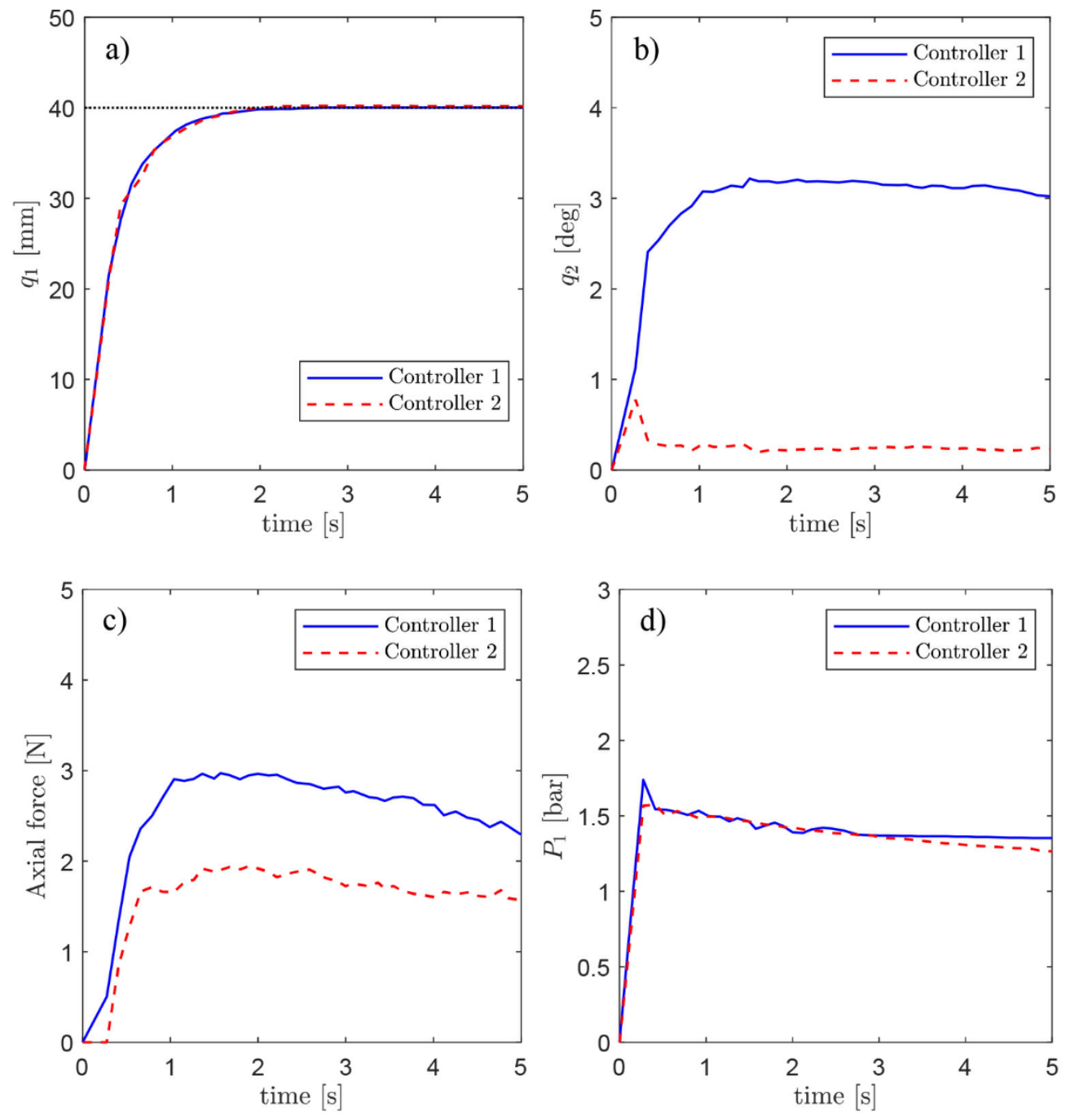

Figure 7. Experimental results on the softer phantom (Shore $A=1)$ for one trial: piston position $(a)$; needle tip rotation (b); axial force (c); control pressure $P_{1}(\mathrm{~d})$. Controller 1 denotes the control law (11) with the observer (3), while Controller 2 denotes the control law (17) with the observer (3).

The control laws (11) and (17) and the adaptive law (3) have been implemented on an embedded microcontroller (mbed NHP LPC1768) mounted on a specially designed PCB. The microcontroller provides a pressure setpoint to the valves and acquires data from the force sensor and from the optical encoder. The measurements from the magnetic tracking system are communicated to the microcontroller via serial link employing a Remote Procedure Call protocol (RPC). The parameters of system (2) are $m=0.01 \mathrm{Kg} ; M=0.05 \mathrm{Kg} ; A_{1}=792 \mathrm{~mm}^{2} ; A_{0}=508 \mathrm{~mm}^{2} ; l=0.135 \mathrm{~m} ; k_{t}=$ $0.173 \mathrm{Nm} ; c_{v}=6$. The value of $c_{v}$ has been estimated experimentally by manually deflecting the needle to induce lateral vibrations and by recording the tip rotation over time. The values of $k_{t}$ and $l$ are computed from (1) with $E=120 \mathrm{GPa}, I=0.1 \mathrm{~mm}^{4}, L=0.15 \mathrm{~m}, \gamma_{\theta}=0.9, k_{\theta}=2.4$, which refer to a $18 \mathrm{G}$ titanium needle mounted as a cantilever beam and subject to an axial tip force. The parameters of Controller 1 have been manually tuned to complete the needle insertion during 3 seconds without overshoot: $k_{p}=30 A_{1} ; k_{v}=20 A_{1} ; k_{m}=2 ; \alpha=50 ; P_{0}=2$. The tuning 
parameters for Controller 2 have been chosen to produce a similar response to that of Controller 1 with the softer phantom: $k_{p}=3000 A_{1} ; k_{v}=40 A_{1} ; k_{m}=2 ; \alpha=50 ; P_{0}=2$. The former values verify respectively the inequality $2 \alpha k_{v}>1$ and the inequality $2 \alpha k_{v} M^{2}>1$ (see Proposition 2 and Proposition 3). The difference between the tuning parameters in both controllers is due to the terms $l$ and $\Delta$ in (17) which multiply $k_{p}$ and $k_{v}$. Instead, the friction forces and the pressure $P_{0}$ contribute in the same way to the Controller 1 and Controller 2 thus the same value of $\alpha$ has been used in both cases. In all experiments the phantom was positioned $10 \mathrm{~mm}$ away from the retracted piston thus the prescribed insertion depth is $30 \mathrm{~mm}$, which is representative of our phantom trials for laser ablation of the liver under MRI guidance [1]. Deeper insertions highlight the effect of the surrounding tissue on the needle in terms of a lateral force $F^{\prime} \neq 0$ and will be investigated as part of future work. The prescribed position $q_{1}^{*}=40 \mathrm{~mm}$ is reached in 2.5 seconds in all experiments, thus the insertion speed is approximately $10 \mathrm{~mm} / \mathrm{s}$, which is representative of percutaneous liver interventions [7].
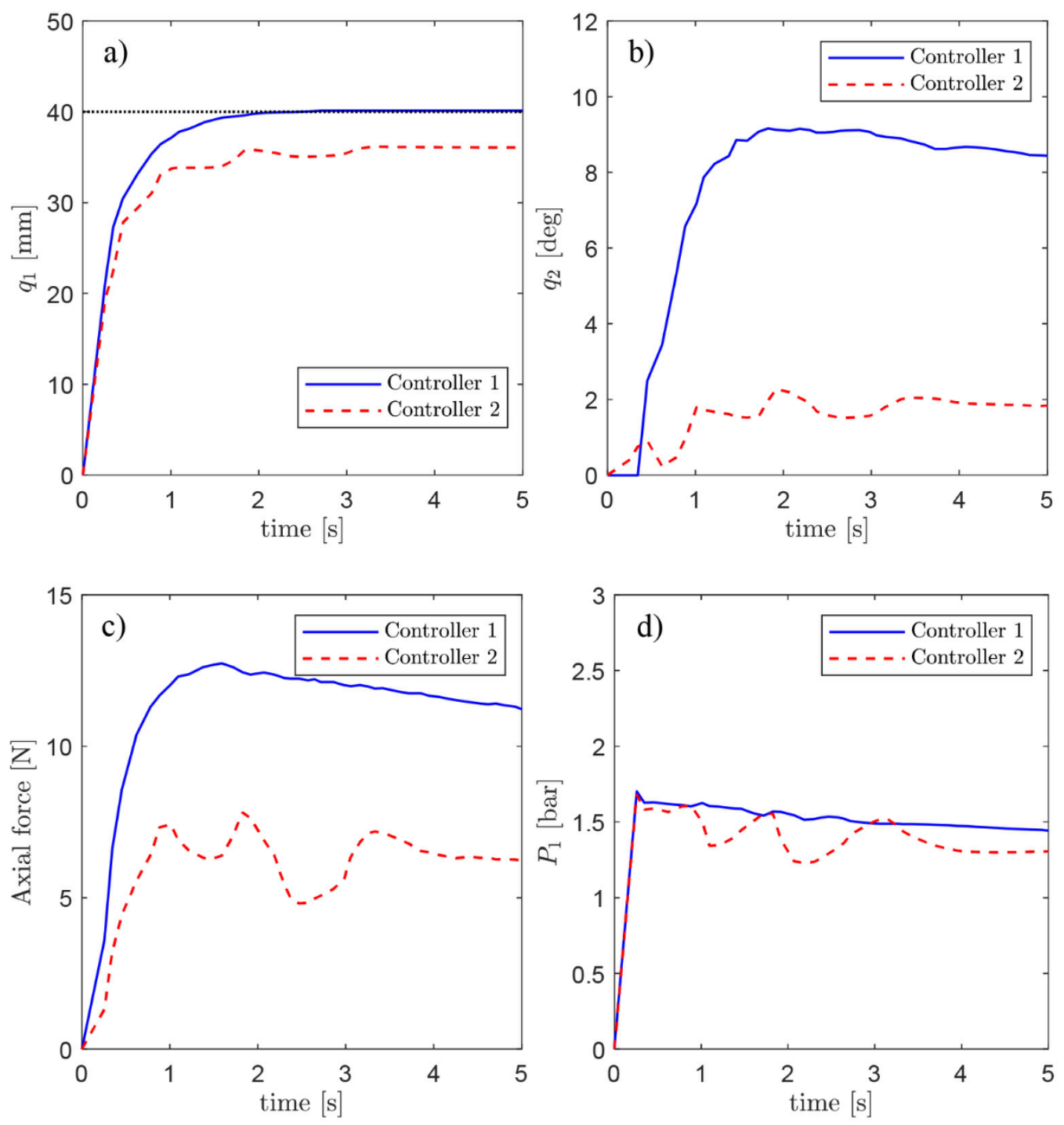

Figure 8. Experimental results on the harder phantom (Shore $A=10$ ) for one trial: piston position (a); needle tip rotation (b); axial force (c); control pressure $P_{1}(\mathrm{~d})$. Controller 1 denotes the control law (11) with the observer (3), while Controller 2 denotes the control law (17) with the observer (3).

The results of the needle insertion in the softer phantom are shown in Figure 7, which refers to one trial. In this case, the system reaches the desired equilibrium $\left(q_{1}^{*}, q_{2}^{*}\right)=(40,0)$ using either Controller 1 or Controller 2. Although the pressure $P_{1}$ is comparable in both cases, the axial force and the tip rotation are clearly lower if Controller 2 is used, which is similar in principle to the simulation results. The tip deflection $\sigma$ of a cantilever beam of length $L$ with tip load can be computed 
from the tip rotation $q_{2}$ as $\sigma=2 L q_{2} / 3$. The final value of the tip deflection with Controller 1 is approximately $5 \mathrm{~mm}$, while the corresponding value with Controller 2 is approximately $0.5 \mathrm{~mm}$. This is a significant difference considering that the lesions normally treated with laser ablation have a minimum diameter of $10 \mathrm{~mm}$ [40], while early diagnosis aims to detect even smaller lesions. The different performance of Controller 1 and Controller 2 is due to the nonlinear function $h(\eta)=\log (\eta)$ employed in (16.a), which modulates the control input depending on the tip rotation $q_{2}$. In addition, the axial force in equation (17) is multiplied by the factor $\cos \left(q_{2}\right)^{2} \leq 1$ thus it decreases with larger $q_{2}$. As observed in the simulations, setting $\alpha=0$ the piston fails to reach the prescribed position with both controllers since the friction forces are not compensated. The cumulative estimate of the friction forces computed with the observer (3) is approximately $15 \mathrm{~N}$, thus its magnitude is comparable to the axial force in this set of experiments.
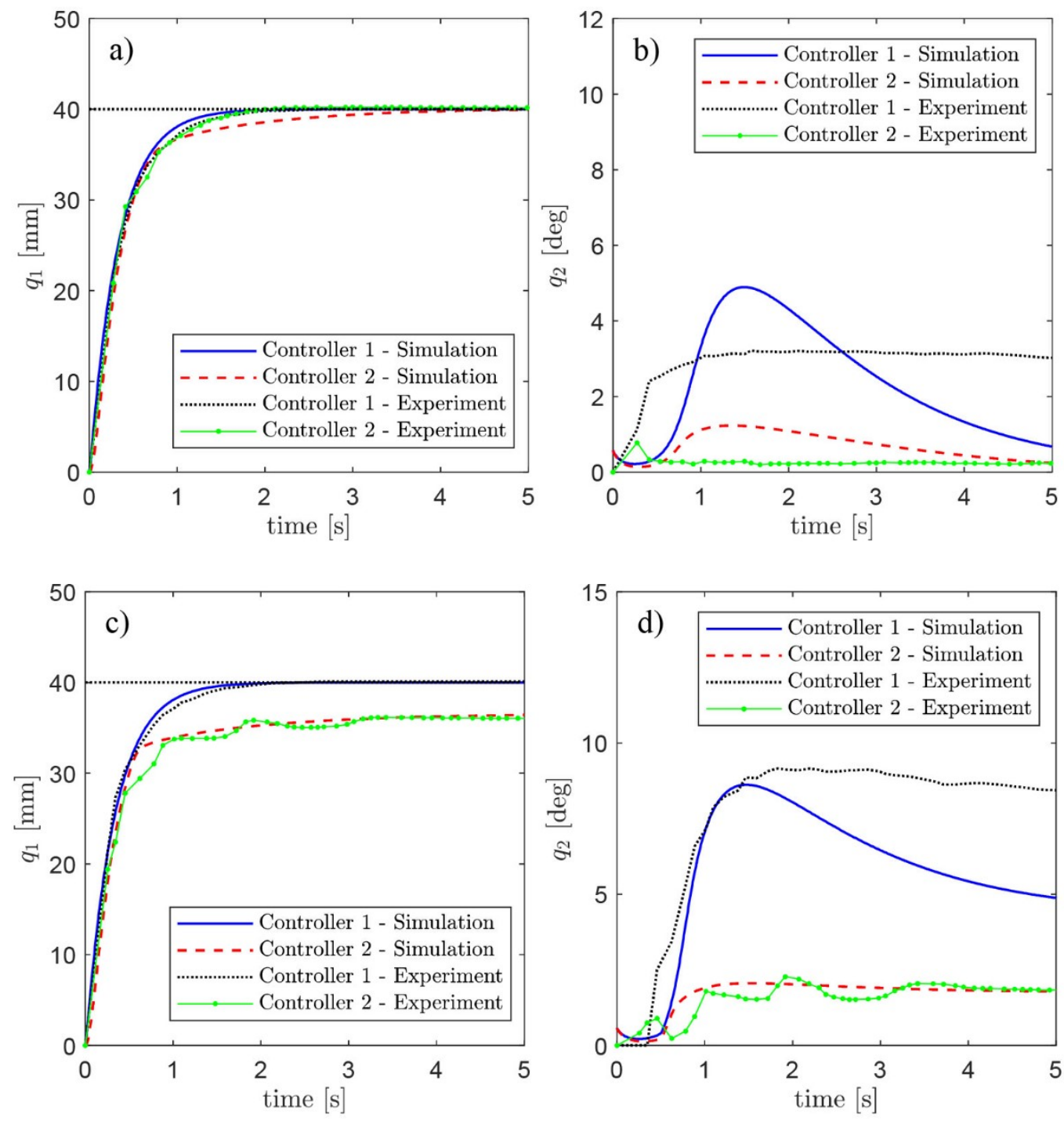

Figure 9. Comparison between simulation results and experimental results: piston position for softer phantom and simulation without lateral force (a); and corresponding needle tip rotation on the bending plane (b); piston position for harder phantom and simulation with a small lateral force $F^{\prime}=$ $F \in(\mathrm{c})$; and corresponding needle tip rotation on the bending plane (d).

The test results for the harder phantom are shown in Figure 8, which refers to one trial. With Controller 1 the piston reaches the prescribed position at the cost of a larger tip rotation. Instead, with Controller 2 the piston stops before reaching the prescribed position at $q_{1}^{\prime}=q_{1}^{*}+\left(\cos \left(q_{2}\right)-1\right) / l=$ $36 \mathrm{~mm}$, but results in an $80 \%$ smaller tip rotation. The final tip deflection $\sigma$ is approximately $14 \mathrm{~mm}$ with Controller 1 and approximately $3 \mathrm{~mm}$ with Controller 2. Comparing the tip rotation at the same 
position $q_{1}=q_{1}^{\prime}=36 \mathrm{~mm}$ shows that $q_{2}=6.6^{\circ}$ with Controller 1 and the corresponding tip deflection is approximately $11.5 \mathrm{~mm}$, while $q_{2}=1.8^{\circ}$ with Controller 2 and the corresponding tip deflection is approximately $3 \mathrm{~mm}$. Thus the tip deflection for this specific system is reduced by more than $70 \%$ with Controller 2. This behaviour is similar in principle to the simulation results in the presence of a small lateral force which suggests that Controller 2 could yield comparable performance for the same type of system and for different experimental setups. In the experiments, the lateral force is applied to the needle by the surrounding material and it is more pronounced with the harder phantom. This point is confirmed prescribing a larger value of $q_{1}^{*}$ corresponding to a deeper insertion: in such a case, the Controller 1 leads to an even larger tip rotation and corresponding deflection, while the Controller 2 yields similar results as those in Figure 8.

For comparison purposes, the simulation results employing the same model parameters for system (2) and the same tuning parameters $k_{p}, k_{v}, k_{m}, \alpha$ as in the experiments are shown in Figure 9 overlaid to the experimental results of Figure 7 and Figure 8 respectively. In addition, the parameters of (23) have been set to $c_{0}=0.5, c_{1}=12, c_{2}=0.25, c_{3}=100 \mathrm{in}$ an attempt to match the response of the real system. To this end the simulation results in Figure 9.c and Figure 9.d also account for a small lateral force $F^{\prime}=F \epsilon$, where $\epsilon=0.002$, that becomes active at $t=1$ second. This suggests that the lateral force in the experiments is approximately two orders of magnitude smaller compared to the axial force and to the friction forces for this specific actuator. Controller 1 and Controller 2 yield qualitatively similar results in simulations and experiments, indicating that the rigid-link model of the needle (2) is appropriate for controller design purposes. Nevertheless, the simulations results are not exactly the same as the experiments because the simplified model (23) of the axial force does not account for the nonlinear stiffness of the phantom. In particular, the discrepancy becomes more noticeable at larger values of $q_{2}$. A possible explanation might be that the model $F^{\prime}=F \epsilon$ does not capture with sufficient accuracy the effect of the lateral forces which are applied by the phantom along the whole length of the needle. Consequently, the first mode shape might not describe the needle deflection with sufficient accuracy in this case. As a result, the needle tip rotation measured in the experiments is generally higher compared to the simulation results.

Increasing $k_{v} / k_{m}$ and reducing $k_{p}$ the movement of the piston becomes slower, which can result in lower axial forces with both controllers. However, a slow needle insertion might be undesirable in clinical practice, as it is the case for percutaneous interventions conducted under breath hold, which must be completed within a few seconds. In addition, the insertion force during the puncture stage is less sensitive to the insertion speed, since it is predominantly dependent on the stiffness of the tissue and on the fracture toughness [41]. Subsequently, the friction between needle and surrounding tissue contributes to the insertion force, thus a lower speed might be particularly beneficial for deeper insertions [7]. Note finally that Controller 1 and Controller 2 employ a constant predefined value of the target position $q_{1}^{*}$ which corresponds to a step command. This approach is commonly used for assessing and comparing the performance of different controllers, but it might not be suitable for clinical use. As part of our future work we intend to introduce a planning algorithm that computes $q_{1}^{*}$ at any instant depending on the system state and on the external commands provided by clinicians, thus resulting in more complex insertion trajectories. Although the proposed controllers do not rely on axial rotation, they could in theory be combined with it. In this respect, axial rotation could become part of the planning algorithms. This approach could help to further reduce the needle tip rotation and the corresponding deflection for deeper insertions and shall be investigated in conjunction with different medical imaging modalities employing a wider range of phantoms.

In summary, the simulation results and the experiments suggest that Controller 2 is preferable to Controller 1 for the softer phantom (Shore $A=1$, representative of breast tissues [39]). The results for the harder phantom (Shore $\mathrm{A}=10$, representative of liver tissues [38]) highlight a trade-off which is related to the underactuated nature of system (2). In particular, Controller 1 achieves the prescribed insertion depth at the cost of a larger needle deflection, while Controller 2 stops the insertion at a shorter depth but reduces the needle deflection. From a theoretical point of view both behaviours are a direct consequence of the proposed energy shaping approach (see Remark 1). From a clinical point of view we believe Controller 2 to be preferable since it corresponds to clinical practice (i.e. manual 
insertions are typically stopped if the needle deflection becomes noticeable) and it could reduce the risks associated to deep insertions with high needle deflection, namely the risk of accidentally puncturing sensitive structures in proximity of the needle path. We intend to investigate this hypothesis in a clinically representative setting as part of our future work.

\section{Conclusions}

This paper presented two adaptive nonlinear controllers for the insertion of a conventional metallic needle with axial-symmetric tip in soft tissues employing a single pneumatic actuator. Differently from established solutions, the proposed approach does not rely on the axial rotation of the needle and does not require additional needle supports. Nevertheless, it could also be used in conjunction with a needle guide or as part of a more complex system that includes axial rotation. While a pneumatic actuator has been chosen in this study, we believe that the proposed solution can be applied to different actuation strategies. An adaptive observer has been designed to estimate the unknown friction forces of the pneumatic actuator without introducing assumptions on their magnitude. A port-Hamiltonian formulation and an energy shaping approach have been employed for the controller design, and the corresponding stability conditions have been expressed for the desired equilibrium. To this end we assumed the lateral force acting on the needle to be zero, which is reasonable for relatively short insertion depths in soft tissue conducted with needles that have an axial-symmetric tip. Nevertheless, the effects of a non-negligible lateral force in terms of stability of the closed-loop equilibrium have been discussed in detail.

Simulations and experiments on a softer silicone rubber phantom showed that with both controllers the piston reaches the required insertion depth with a small needle deflection. This result suggests that the lateral forces are considerably smaller than the axial force in this particular case, as initially hypothesized. Instead, the experiments on a harder phantom highlight the detrimental effects of the lateral forces which are not negligible in this case, as also confirmed by the simulation results. In particular, the first controller allows reaching the required insertion depth at the cost of a larger tip rotation. Instead, the second controller allows reducing the needle tip rotation by modulating the axial force and, in case of harder phantoms, also by limiting the insertion depth. This behaviour might serve the purpose of preventing deep insertions with large deflection thus it could help to reduce the risk of puncturing sensitive structures, such as blood vessels, located in proximity of the insertion path, and it could be used in conjunction with different needle-insertion robots. Confirming this hypothesis in a clinically representative setting is part of our future work. To this end, we intend to investigate the use of advanced planning algorithms, which we expect will bring the largest improvement in performance within clinical settings. We shall study this aspect in conjunction with medical imaging modalities. Finally, we aim to investigate the effect of lateral forces in the context of deeper insertions and to validate our results with extensive testing on a wider range of phantoms considering different insertion speeds.

\section{Acknowledgements}

This research was supported by the Engineering and Physical Sciences Research Council (grant number EP/R009708/1). Alessandro Astolfi is also supported by the European Union's Horizon 2020 Research and Innovation Programme under grant agreement No 739551 (KIOS CoE).

\section{References}

[1] E. Franco, D. Brujic, M. Rea, W.M. Gedroyc, M. Ristic, Needle-Guiding Robot for Laser Ablation of Liver Tumors Under MRI Guidance, IEEE/ASME Trans. Mechatronics. 21 (2016) 931-944. https://doi.org/10.1109/TMECH.2015.2476556.

[2] D. Stoianovici, C. Kim, G. Srimathveeravalli, P. Sebrecht, D. Petrisor, J. Coleman, S.B. Solomon, H. Hricak, MRI-safe robot for endorectal prostate biopsy, IEEE/ASME Trans. Mechatronics. 19 (2014) 1289-1299.

[3] O. Piccin, B. Bayle, B. Maurin, M. de Mathelin, Kinematic modeling of a 5-DOF parallel mechanism for semi-spherical workspace, Mech. Mach. Theory. 44 (2009) 1485-1496. 
https://doi.org/10.1016/j.mechmachtheory.2008.11.012.

[4] W. Ye, B. Zhang, Q. Li, Design of a 1R1T planar mechanism with remote center of motion, Mech. Mach. Theory. 149 (2020) 103845.

https://doi.org/10.1016/j.mechmachtheory.2020.103845.

[5] M. Moche, S. Heinig, N. Garnov, J. Fuchs, T.-O. Petersen, D. Seider, P. Brandmaier, T. Kahn, H. Busse, Navigated MRI-guided liver biopsies in a closed-bore scanner: experience in 52 patients, Eur. Radiol. 26 (2015) 2462-2470. https://doi.org/10.1007/s00330-015-40971.

[6] E. Franco, M. Ristic, M. Rea, W.M.W. Gedroyc, Robot-assistant for MRI-guided liver ablation: A pilot study, Med. Phys. 43 (2016) 5347-5356. https://doi.org/10.1118/1.4961986.

[7] D.J. van Gerwen, J. Dankelman, J.J. van den Dobbelsteen, Needle-tissue interaction forces--a survey of experimental data., Med. Eng. Phys. 34 (2012) 665-80. https://doi.org/10.1016/j.medengphy.2012.04.007.

[8] A. Sakes, D. Dodou, P. Breedveld, Buckling prevention strategies in nature as inspiration for improving percutaneous instruments: a review, Bioinspir. Biomim. 11 (2016) 021001. https://doi.org/10.1088/1748-3190/11/2/021001.

[9] N.J. van de Berg, D.J. van Gerwen, J. Dankelman, J.J. van den Dobbelsteen, Design Choices in Needle Steering - A Review, IEEE/ASME Trans. Mechatronics. 20 (2015) 2172-2183. https://doi.org/10.1109/TMECH.2014.2365999.

[10] N. Abolhassani, R. V. Patel, F. Ayazi, Minimization of needle deflection in robot-assisted percutaneous therapy, Int. J. Med. Robot. Comput. Assist. Surg. 3 (2007) 140-148. https://doi.org/10.1002/rcs.136.

[11] B. Fallahi, M. Waine, C. Rossa, R. Sloboda, N. Usmani, M. Tavakoli, An IntegratorBackstepping Control Approach for 3D Needle Steering, IEEE/ASME Trans. Mechatronics. (2019) 1-1. https://doi.org/10.1109/TMECH.2019.2930732.

[12] T. Watts, R. Secoli, F.R. y Baena, A Mechanics-Based Model for 3-D Steering of Programmable Bevel-Tip Needles, IEEE Trans. Robot. 35 (2018) 1-16. https://doi.org/10.1109/TRO.2018.2879584.

[13] M. Scali, P. Breedveld, D. Dodou, Experimental evaluation of a self-propelling bio-inspired needle in single- and multi-layered phantoms, Sci. Rep. 9 (2019) 1-13. https://doi.org/10.1038/s41598-019-56403-0.

[14] S.C. Ryu, Z.F. Quek, J.-S. Koh, P. Renaud, R.J. Black, B. Moslehi, B.L. Daniel, K.-J. Cho, M.R. Cutkosky, Design of an Optically Controlled MR-Compatible Active Needle, IEEE Trans. Robot. 31 (2015) 1-11. https://doi.org/10.1109/TRO.2014.2367351.

[15] H.B. Gilbert, J. Neimat, R.J. Webster, Concentric Tube Robots as Steerable Needles: Achieving Follow-the-Leader Deployment, IEEE Trans. Robot. 31 (2015) 246-258. https://doi.org/10.1109/TRO.2015.2394331.

[16] C. Yang, Y. Xie, S. Liu, D. Sun, Force Modeling, Identification, and Feedback Control of Robot-Assisted Needle Insertion: A Survey of the Literature, Sensors. 18 (2018) 561. https://doi.org/10.3390/s18020561.

[17] B. Fallahi, C. Rossa, R.S. Sloboda, N. Usmani, M. Tavakoli, Sliding-based image-guided 3D needle steering in soft tissue, Control Eng. Pract. 63 (2017) 34-43. https://doi.org/10.1016/J.CONENGPRAC.2017.04.001.

[18] D.C. Rucker, J. Das, H.B. Gilbert, P.J. Swaney, M.I. Miga, N. Sarkar, R.J. Webster, Sliding Mode Control of Steerable Needles, IEEE Trans. Robot. 29 (2013) 1289-1299. https://doi.org/10.1109/TRO.2013.2271098.

[19] P. Moreira, S. Misra, Biomechanics-Based Curvature Estimation for Ultrasound-guided Flexible Needle Steering in Biological Tissues, Ann. Biomed. Eng. 43 (2015) 1716-1726. https://doi.org/10.1007/s10439-014-1203-5.

[20] J. Moreno-Valenzuela, C. Aguilar-Avelar, S. Puga-Guzman, On trajectory tracking control of the inertia wheel pendulum, in: 2014 Int. Conf. Control. Decis. Inf. Technol., IEEE, 2014: pp. 572-577. https://doi.org/10.1109/CoDIT.2014.6996958. 
[21] R. Tsumura, J.S. Kim, H. Iwata, I. Iordachita, Preoperative Needle Insertion Path Planning for Minimizing Deflection in Multilayered Tissues, IEEE Robot. Autom. Lett. 3 (2018) 2129-2136. https://doi.org/10.1109/LRA.2018.2809540.

[22] M. Motaharifar, H.A. Talebi, F. Abdollahi, A. Afshar, Nonlinear Adaptive Output-Feedback Controller Design for Guidance of Flexible Needles, IEEE/ASME Trans. Mechatronics. 20 (2015) 1912-1919. https://doi.org/10.1109/TMECH.2014.2359181.

[23] A.D.R. Li, J. Plott, L. Chen, J.S. Montgomery, A. Shih, Needle deflection and tissue sampling length in needle biopsy, J. Mech. Behav. Biomed. Mater. 104 (2020) 103632. https://doi.org/10.1016/j.jmbbm.2020.103632.

[24] E. Franco, T. Brown, Energy Shaping Control for Robotic Needle Insertion, in: 23rd Int. Conf. Syst. Theory, Control Comput., Sinaia, Romania, 2019: pp. 1-6. https://doi.org/10.1109/ICSTCC.2019.8886055.

[25] A. Astolfi, R. Ortega, Immersion and invariance: A new tool for stabilization and adaptive control of nonlinear systems, IEEE Trans. Automat. Contr. 48 (2003) 590-606. https://doi.org/10.1109/TAC.2003.809820.

[26] A. Astolfi, D. Karagiannis, R. Ortega, Nonlinear and Adaptive Control with Applications, Berlin, 2007. https://doi.org/10.1007/978-1-84800-066-7.

[27] E. Franco, M. Rea, W. Gedroyc, M. Ristic, Control of a Master-Slave Pneumatic System for Teleoperated Needle Insertion in MRI, IEEE/ASME Trans. Mechatronics. 21 (2016) 25952600. https://doi.org/10.1109/TMECH.2016.2577608.

[28] E. Franco, A. Astolfi, F. Rodriguez y Baena, Robust balancing control of flexible invertedpendulum systems, Mech. Mach. Theory. 130 (2018) 539-551. https://doi.org/10.1016/J.MECHMACHTHEORY.2018.09.001.

[29] S.Y. Ko, F. Rodriguez y Baena, Toward a miniaturized needle steering system with path planning for obstacle avoidance., IEEE Trans. Biomed. Eng. 60 (2013) 910-7. https://doi.org/10.1109/TBME.2012.2227741.

[30] F. Galassi, D. Brujic, M. Rea, N. Lambert, N. Desouza, M. Ristic, Fast and accurate localization of multiple RF markers for tracking in MRI-guided interventions, Magn. Reson. Mater. Physics, Biol. Med. 28 (2014) 33-48. http://link.springer.com/article/10.1007\%2Fs10334-014-0446-3.

[31] E. Franco, M. Ristic, Adaptive control of a master-slave system for teleoperated needle insertion under MRI-guidance, in: 2015 23rd Mediterr. Conf. Control Autom., IEEE, 2015: pp. 61-67. https://doi.org/10.1109/MED.2015.7158730.

[32] L.L. Howell, A. Midha, T.W. Norton, Evaluation of Equivalent Spring Stiffness for Use in a Pseudo-Rigid-Body Model of Large-Deflection Compliant Mechanisms, J. Mech. Des. 118 (1996) 126. https://doi.org/10.1115/1.2826843.

[33] Y.-Q. Yu, L.L. Howell, C. Lusk, Y. Yue, M.-G. He, Dynamic Modeling of Compliant Mechanisms Based on the Pseudo-Rigid-Body Model, J. Mech. Des. 127 (2005) 760. https://doi.org/10.1115/1.1900750.

[34] F.M. Foong, C.K. Thein, D. Yurchenko, On mechanical damping of cantilever beam-based electromagnetic resonators, Mech. Syst. Signal Process. 119 (2019) 120-137. https://doi.org/10.1016/j.ymssp.2018.09.023.

[35] R. Ortega, M.W. Spong, F. Gomez-Estern, G. Blankenstein, Stabilization of a class of underactuated mechanical systems via interconnection and damping assignment, IEEE Trans. Automat. Contr. 47 (2002) 1218-1233. https://doi.org/10.1109/TAC.2002.800770.

[36] E. Franco, Adaptive IDA-PBC for underactuated mechanical systems with constant disturbances, Int. J. Adapt. Control Signal Process. 33 (2019) 1-15. https://doi.org/10.1002/acs.2947.

[37] A.M. Okamura, C. Simone, M.D. O'Leary, Force modeling for needle insertion into soft tissue., IEEE Trans. Biomed. Eng. 51 (2004) 1707-16. https://doi.org/10.1109/TBME.2004.831542.

[38] Y.C. Yoon, J.S. Lee, S.U. Park, J.H. Kwon, T.H. Hong, D.G. Kim, Quantitative assessment 
of liver fibrosis using shore durometer, Ann. Surg. Treat. Res. 93 (2017) 300-304. https://doi.org/10.4174/astr.2017.93.6.300.

[39] C. Ashlie Davis, K. Lynn Mittura, G. Elizabeth Copeland, E. Martina Hawkins, C. Ashlie, K. Lynn, G. Elizabeth, E. Martina, Feasibility of a touch sensitive breast phantom for use in the training of physicians in clinical breast examination, 2011.

[40] A.L. Gough-Palmer, W.M.W. Gedroyc, Laser ablation of hepatocellular carcinoma-A review, World J. Gastroenterol. 14 (2008) 7170-7174.

[41] M. Khadem, C. Rossa, N. Usmani, R.S. Sloboda, M. Tavakoli, A Two-body Rigid/Flexible Model of Needle Steering Dynamics in Soft Tissue, IEEE/ASME Trans. Mechatronics. PP (2016) 1-1. https://doi.org/10.1109/TMECH.2016.2549505. 


\section{Appendix}

The matrices $G, G^{\perp}, D, \mathcal{M}$, the potential energy $V$, and the external forces $\delta$ for system (2) are given by the equations

$$
\begin{array}{cc}
G=A_{1}\left[\begin{array}{l}
1 \\
0
\end{array}\right], \quad G^{\perp}=\left[\begin{array}{ll}
0 & 1
\end{array}\right], & D=\left[\begin{array}{ll}
0 & 0 \\
0 & c_{v}
\end{array}\right], \quad V=\frac{k_{t}}{2} q_{2}^{2}, \\
\mathcal{M}=\left[\begin{array}{cc}
M+m & -m l \sin \left(q_{2}\right) \\
-m l \sin \left(q_{2}\right) & m l^{2}
\end{array}\right], \quad \delta=\frac{F}{\Delta}\left[\begin{array}{c}
\cos \left(q_{2}\right)^{2} \\
-\frac{M \sin \left(q_{2}\right)}{m l}
\end{array}\right]-\frac{F^{\prime}}{\Delta}\left[\begin{array}{c}
\sin \left(q_{2}\right) \cos \left(q_{2}\right) \\
\frac{(M+m) \cos \left(q_{2}\right)}{m l}
\end{array}\right] .
\end{array}
$$

The matrices $G^{\prime}, G^{\prime \perp}, D^{\prime}, \mathcal{M}^{\prime}$, and the potential energy $V^{\prime}$ after the partial feedback linearization (15) are given by the equations

$$
\begin{gathered}
G^{\prime}=A_{1}\left[\frac{\sin \left(q_{2}\right)}{l}\right], \quad G^{\perp}=\left[\begin{array}{cc}
\frac{\sin \left(q_{2}\right)}{l} & -1
\end{array}\right], \\
D^{\prime}=\left[\begin{array}{cc}
0 & 0 \\
0 & \frac{c_{v}}{m l^{2}}
\end{array}\right], \quad V^{\prime}=\frac{k_{t}}{2 m l^{2}} q_{2}^{2}, \quad \mathcal{M}^{\prime}=I .
\end{gathered}
$$

